\title{
Trayectorias tempranas en el inicio de la vida adulta en México*
}

\author{
Guadalupe Fabiola Pérez Baleón**
}

En este artículo se examinan las trayectorias en el inicio a la vida adulta de tres cohortes de mujeres y hombres mexicanos. Se precisa una mayor diversidad de secuencias en las mujeres, dadas por el incremento de su escolaridad y participación laboral y un acotamiento en las trayectorias de los varones en torno a dos trayectorias. Los resultados permiten confirmar la existencia de normatividades de género presentes entre hombres y mujeres pero también entre las propias mujeres y entre los mismos varones. Esta normatividad se entreteje con otras desigualdades, como las socioeconómicas, para producir trayectorias más comunes a ciertos grupos sociales y a determinados lugares y tiempos.

Palabras clave: curso de vida, género, cohortes, transiciones, trayectorias.

Fecha de recepción: 22 de junio de 2012.

Fecha de aceptación: 14 de agosto de 2013.

\section{Early Trajectories in Initial Adult Life in Mexico}

The article examines the trajectories most common in the configuration of the adult life of three cohorts of Mexican men and women. It can be established a greater diversity of sequence in women given by the increase of their schooling and labor participation. Men were concentrated around two trajectories. The results confirmed the existence of gender regulations present between men and women but also among women and among men. This regulation is interconnected with socio-economic inequalities to produce trajectories that are more characteristic to certain social groups and determinate time and place.

Key words: life course, gender, cohort, transitions, trajectories.

* Agradezco a Olga Rojas, Marta Mier y Terán y Orlandina de Oliveira su lectura y comentarios a la versión original de este artículo. También reconozco el apoyo de Nina Castro y Ángeles Sánchez Bringas en la discusión de este trabajo, y agradezco las observaciones de los dos lectores anónimos que revisaron el manuscrito. Este artículo fue realizado durante mi estancia posdoctoral en la UAM-Xochimilco.

** Posdoctorante en la Universidad Autónoma Metropolitana-Xochimilco, División de Ciencias Sociales y Humanidades, Departamento de Política y Cultura. Correo electrónico:<gfperez@correo.xoc.uam.mx>y <ggfabiola@hotmail.com>. 


\section{Introducción}

Este trabajo tiene como objetivo principal profundizar en el cambio inter e intra cohorte de las secuencias más comunes que se conforman a partir de la ocurrencia de la salida de la escuela, la primera unión conyugal y el primer trabajo de mujeres y varones mexicanos nacidos en tres momentos del siglo Xx. Conforme a una perspectiva de curso de vida se analizan la temporalidad y la duración de las transiciones que conforman dichas trayectorias, así como el intervalo entre el inicio y el fin de aquellas más comunes para cada uno. Aunque es posible apreciar tendencias generales en el país, se considera que las secuencias responden a la par que cristalizan la normatividad social de cierto lugar, tiempo y grupo social en torno al momento y el orden en que los jóvenes deben transitar hacia la adultez. Las trayectorias, desde este punto de vista, son una síntesis de un complejo proceso dinámico, en donde múltiples fuerzas estructurales han influido en la vida de las personas, a la vez que han sido influidas por ellas, teniendo como eje -no el único pero sí uno de los más importantes- al género, el cual atraviesa permanentemente a la sociedad en varias direcciones y se interrelaciona con otras desigualdades, con efectos perdurables en el tiempo.

El género opera como un estándar implícito de normalización y brinda los parámetros de la acción que rigen, determinando "lo que aparecerá y no aparecerá en la esfera de lo social” (Butler, 2006: 69, en Sánchez, 2013). La normatividad de género condensa los significados, las ideaciones, las nociones y los valores de diferentes discursos culturales en un saber predominante, aunque nunca homogéneo o monolítico, sobre lo que es ser "hombre" o "mujer". Las acciones individuales "citan" estos significados actuándolos cotidianamente en varias formas, aunque nunca reproduciéndolos de manera idéntica, lo que da lugar a la heterogeneidad, al conflicto y a la presencia en la escena social de comportamientos que se salen de la norma (Sánchez, 2013).

Además del género, los calendarios varían por nivel socioeconómico, reflejando diferencias culturales generalizadas en la estratificación social (Hogan y Astone, 1986). Tilly (2000) afirma que las distinciones categoriales que predominan en una sola organización social ganan en fortaleza y disminuyen los costos de mantener la desigualdad, en este caso económica, cuando se asocian a categorías pareadas y desiguales ampliamente accesibles, mismas que ya regían en el total de la sociedad, tales como las desigualdades de género. Cuando muchas organizaciones adoptan las mismas distinciones categoriales, éstas al- 
canzan mayor difusión y son más decisivas en la vida social en general, contribuyendo a su persistencia en el tiempo y dentro de los distintos ámbitos sociales. Lo anterior pone de manifiesto que los miembros de una cohorte no están uniformemente expuestos al cambio.

Por tal, es indispensable realizar estudios que contemplen dichas diferenciaciones, con categorías que incluyan el nivel socioeconómico, el género, la residencia rural o urbana, la etnicidad y el nivel educativo de las personas, a fin de dar cuenta sobre las variaciones sociales dentro de una misma cohorte y entre cohortes (Elder, 1975; Elder, Kirkpatrick y Crosnoe, 2003).

Se parte de la idea de que en un país tan diverso y contrastante como México, más que un modelo unitario al estilo de las naciones desarrolladas, existen trayectorias que pueden ser más comunes a ciertos grupos que a otros, derivadas de la normatividad del entorno en que éstos se desarrollan y en función de las desigualdades de género y económicas, así como de los cambios históricos que a cada cohorte le tocó vivir, por lo que es factible precisar más de una trayectoria en cada cohorte. Lo interesante entonces es determinar esas otras trayectorias, develar cómo el género y el nivel socioeconómico han dejado su impronta en su conformación, y precisar qué tan constantes o diferenciales han sido estas trayectorias en el tiempo.

Para tener una visión general de cómo el género, el nivel socioeconómico y las cohortes de nacimiento han ido conformando estas trayectorias se organizó el presente texto en cuatro apartados. En el primero se presenta una revisión de los estudios sociodemográficos mexicanos que han abordado diferentes transiciones del curso de vida y las han ordenado construyendo tipologías. Se hace especial énfasis en los artículos que bajo el enfoque de curso de vida presentan trayectorias integradas con una o más de las transiciones que aquí se abordan y estiman de manera similar; con ello se ha tratado de establecer sus avances, en conjunto con los temas y preguntas que quedan sin resolver, y a los cuales este artículo pretende abonar.

En el segundo apartado se analizan las principales trayectorias que han estado presentes a lo largo de seis décadas. Aquí el acento está puesto en los cambios en el tiempo, captados por medio de las cohortes de nacimiento. En el tercero se estudia la temporalidad de las secuencias más comunes a estas tres cohortes, mismas que son diferenciales entre hombres y mujeres, lo que permite observar la cristalización de los mandatos de género en la conformación de la secuencia, la velocidad y la temporalidad de estas trayectorias. 
En el cuarto apartado se prueba, mediante modelos de tiempo discreto, la asociación del nivel socioeconómico, la cohorte de nacimiento, el género y el lugar de residencia en la configuración de las trayectorias que iniciaron con la salida de la escuela, versus aquellas que no lo hicieron. Con estos modelos se confirman las tendencias por cohorte y género observadas en los apartados dos y tres, a la vez que se precisa la interrelación entre el sexo, el nivel socioeconómico y el lugar de residencia en la transición hacia la adultez.

En este artículo se utiliza el término "trayectorias tempranas" para referirse a los ordenamientos que surgen durante el periodo de la niñez y concluyen en la juventud media, 29 años, dados por la combinación de tres transiciones; en este caso la salida de la escuela, ${ }^{1}$ la primera unión conyugal ${ }^{2}$ y el primer trabajo estable, ${ }^{3}$ cualquiera que sea su orden, mismos que contribuyen a configurar el inicio de la vida adulta. En este ejercicio se construyeron trayectorias para tres cohortes de mujeres y de hombres mexicanos y se establece la secuencia, la duración ${ }^{4}$ y la temporalidad de las transiciones que conforman las trayectorias más comunes. Tales ordenamientos determinan en gran medida el presente y el futuro de las personas, a la vez que sintetizan las desigualdades socioeconómicas y de género a las que se han enfrentado los miembros de cada cohorte.

\section{El estudio de las trayectorias en México}

La literatura sobre el curso de vida se ha venido incrementando cada vez más en México. ${ }^{5}$ Dos de los temas en que más se ha ahondado son las transiciones a la adultez y las trayectorias vitales de hombres y mu-

${ }^{1}$ Ésta se puede presentar por egreso escolar o por abandono de los estudios, con connotaciones sociales diferenciales según el motivo de salida. La base de datos empleada no permite distinguir los motivos por los cuales la persona finalizó sus estudios, por ello se decidió denominarla en su forma más amplia como la salida de la escuela.

${ }^{2}$ Se hace referencia a la primera unión conyugal ya que la fuente de datos usada no permite distinguir si ésta fue de tipo religiosa y/o civil o consensual, por lo que dentro de esta categoría se abarcan dichos estados civiles.

${ }^{3}$ Debido a que la encuesta empleada captó el trabajo extradoméstico sólo si éste había durado al menos un año, se tomó la decisión de denominarlo como trabajo estable, en contraposición con aquel que duró unos cuantos meses y que por tanto no pudo ser reportado en la encuesta.

${ }^{4}$ El tiempo transcurrido entre una transición y otra conforma la duración del evento que las separa (Ariza, 2000: 179).

${ }^{5}$ Blanco (2002 y 2011) presenta a detalle un panorama de las investigaciones sociodemográficas que sobre curso de vida se han realizado en México. 
jeres. Ambos conceptos ofrecen un panorama diferencial y a la vez complementario de un tema determinado al permitir observar un proceso longitudinal común en donde las primeras dan idea del corto alcance y están imbricadas en las trayectorias, dándoles formas y significados distintivos, mientras que las segundas, marcadas por una secuencia de eventos vitales y transiciones, se orientan al largo plazo (Elder, 1985: 31; Ariza, 2000: 179).

Algunos trabajos sobre trayectorias estudian las carreras o itinerarios que las personas siguen en determinado dominio institucional ${ }^{6}$ En este tipo de estudios el acento está puesto única o primordialmente en un ámbito de la vida, del cual se observan las distintas transiciones y/o estados que la conforman. En este sentido, la línea de investigación en que los estudios sociodemográficos mayormente han profundizado ha sido la referente a las trayectorias laborales. En ellas se han precisado las entradas y salidas del sector formal e informal del mercado laboral de hombres y mujeres (Pacheco y Parker, 2001), se ha estudiado la movilidad intrageneracional de unos y otras dada por el paso de un tamaño de empresa a otro (Coubès, 2005) o se ha privilegiado la clasificación de las ocupaciones para determinar los patrones de movilidad ocupacional de los varones regiomontanos (Solís y Billari, 2003).

Dada la interdependencia que guarda el ámbito familiar conyugal y reproductivo con el laboral en la vida de las mujeres, algunos estudios presentan aproximaciones a dicha relación a partir del concepto de trayectoria, tal como los artículos de Suárez (1992), Blanco (2001 y 2002), Blanco y Pacheco (2001 y 2003) y Ariza y Oliveira (2005). Si bien ha predominado el análisis de las trayectorias laborales, también es posible encontrar estudios donde la trayectoria que prima es la conyugal, y a partir de ella se observa su imbricación con las demás (Quilodrán, 1996).

En tales análisis se reporta, por medio de itinerarios o tipologías cuantitativas o cualitativas, la mayor discontinuidad que las trayectorias laborales femeninas guardan en relación con las masculinas, como resultado de la especialización de las mujeres en las labores reproductivas. Esto es producto de las densas e históricas relaciones entre los

${ }^{6}$ Los dominios se refieren a una esfera institucional, a un campo de actividad, pertenencia, membresía o participación dentro del cual los individuos pueden ser observados en cualquier momento en el tiempo. Algunos dominios institucionales del curso de vida incluyen la escuela, el trabajo, la familia, el hogar y la residencia (Tuirán, 2001: 212). 
sexos que se han establecido sobre una base biológica que naturaliza, perpetúa y legitima las desigualdades construidas en comunidad como resultado del tipo de organización social y de género, así como de las relaciones de reproducción social que prevalecen en determinado tiempo y lugar, las cuales a pesar de su aparente inmutabilidad pueden ser modificadas por la misma interacción sociocultural (Scott, 1996; Lagarde, 1996).

Asimismo los autores han construido las trayectorias en una segunda forma, combinando la ocurrencia de tres o más transiciones y/o eventos particulares que pertenecen a distintos dominios institucionales, algunos de los cuales -aunque no todos- ocurren por primera vez. En estos estudios es común presentar las secuencias resultantes de las transiciones analizadas a fin de establecer aquellas más comunes o típicas en el paso a la vida adulta (Tuirán, 2001; Echarri y Pérez Amador, 2007; Oliveira y Mora, 2008), en la corresidencia y conformación del núcleo familiar cercano (Echarri, 2005), en la formación de uniones conyugales y el establecimiento de arreglos residenciales (Mier y Terán, 2012), en la constitución familiar y el uso por primera vez de la anticoncepción (Brugeilles y Samuel, 2005), en las tendencias de la práctica anticonceptiva (Brugeilles, 2005), en la ocurrencia de la migración y de los eventos con los cuales se relaciona inmediatamente: trabajo, escolaridad y matrimonio (Ariza, 2000), y en la presencia de la migración previa a la vida familiar (Sebille, 2005). Otros más buscan conocer si se cumple el modelo normativo que se esperaría de ciertas transiciones (Tuirán, 2001; Coubés y Zenteno, 2005; Solís, Gayet y Juárez, 2008).

Para determinar las preguntas centrales y los retos de investigación que este trabajo pretende resolver se revisaron con más detalle siete disertaciones con abordajes teóricos y metodológicos similares a éste. Dada la variedad de temáticas que los trabajos antes mencionados abordan, se decidió seleccionar exclusivamente a aquellos que cumplieran dos condiciones: 1) que estudiaran una, dos o las tres transiciones aquí expuestas, y 2) que en la construcción de las secuencias se hubiera seguido un procedimiento similar al de este artículo.

Es posible encontrar una serie de trabajos sobre trayectorias donde la primera unión es el eje de los análisis; entre ellos destaca la investigación sobre noviazgo, unión conyugal y arreglos residenciales por género, generación y estratos socioeconómicos realizada por Mier y Terán (2012) para mujeres y hombres mexicanos nacidos entre 1921 y 1975, basada en la Encuesta Nacional sobre la Dinámica de las Familias (Endifam, 2005). En este estudio se examinan el proceso de for- 
mación de la pareja conyugal y la adquisición de la autonomía respecto de la autoridad familiar. La autora calcula la edad en que ocurrieron las tres transiciones, así como los factores que influyeron en cada una. Busca además conocer la manera y las condiciones en las que las tres transiciones se entrelazan, por lo que propone ocho trayectorias que resultan de la combinación de la ocurrencia o no de un noviazgo corto, una unión temprana y la permanencia en el hogar paterno para todos los que ya habían vivido alguna vez en una relación de pareja. De entre las trayectorias más representativas, obtenidas mediante proporciones, destaca aquella donde la pareja tuvo noviazgos largos, se unió a edades no tempranas y formó un hogar con residencia independiente, así como la secuencia opuesta dada por noviazgos cortos, uniones tempranas y permanencia en el hogar paterno.

En una línea de análisis similar se inscribe el estudio realizado por Echarri (2005) en el que busca dar cuenta de las trayectorias seguidas por las cohortes de mujeres y de varones de la Encuesta Demográfica Retrospectiva (Eder, 1998) en la constitución de la familia y la corresidencia de las personas con sus padres, cónyuge e hijos. Para ello genera ocho situaciones familiares para cada cohorte y sexo en las que interrelaciona la salida del hogar paterno, la entrada en unión y el nacimiento de los hijos, y presenta la distribución de la población según la situación familiar de los individuos a los 30 años, así como el tiempo que duraron en cada situación y la edad mediana de tales transiciones.

Entre los resultados encontrados destaca el hecho de que la mayoría de las personas incluidas en el estudio ya habían conformado un hogar independiente de sus padres y habían iniciado la reproducción, con intervalos cortos entre la unión y la descendencia al momento del corte. Otras secuencias observadas fueron aquellas donde la persona, sin distinción de sexo, aún residía con sus padres a la edad de 30 años, tuviera o no pareja marital e hijos, situación que puede tener su explicación en el alargamiento del periodo de la vida dependiente de los padres, especialmente en el caso de las mujeres.

El interés por conocer el orden de otros eventos una vez que hombres y mujeres han comenzado su vida en pareja llevó a Brugeilles y Samuel (2005) a examinar qué tanto la secuencia de la primera unión, el nacimiento del primer hijo y el uso por primera vez de la anticoncepción han sido una norma para la mayoría de los mexicanos, o si se ha dado una diversidad de trayectorias a lo largo de las tres cohortes que conforman la Eder 1988. Al combinar los tres eventos definieron 15 com- 
binaciones, y mediante la distribución de la población según las secuencias de constitución familiar encontraron que a pesar de las múltiples posibilidades el inicio de la vida familiar comienza en su gran mayoría, y en ambas poblaciones, con la formación de la pareja, el nacimiento de al menos un hijo en un lapso promedio de dos años, y la eventual regulación de su fecundidad. Más que por género, precisan diferencias por cohorte, ya que en la más antigua la trayectoria que dominó fue la unión y el primer hijo, en tanto que en la intermedia comenzaron a usarse los métodos anticonceptivos luego de haberse presentado las otras dos transiciones. Este trayecto se acentuó aún más en la generación joven, además de que surgieron itinerarios en donde comenzaron a disociarse el matrimonio de la procreación al emplearse los métodos anticonceptivos para retardar la llegada del primer hijo.

Otro trabajo enfocado en el estudio de la primera unión marital, así como en la temporalidad y el ordenamiento de la primera relación sexual y el embarazo, fue el realizado por Solís, Gayet y Juárez (2008), quienes estudiaron tres cohortes de mujeres nacidas entre 1951 y 1975 captadas por la Encuesta Nacional sobre Niveles de Vida de los Hogares (Ennvih, 2002). Su intención fue determinar si se había alterado en el tiempo la secuencia normativa o tradicional dada por el primer enlace conyugal, la ocurrencia de la primera relación sexual y del primer embarazo. Además de calcular los cuartiles de edad de estas transiciones presentaron, mediante distribución porcentual, ocho secuencias producto de la combinación de las tres transiciones, y encontraron que la trayectoria normativa pareció haber cedido lugar a otro tipo de trayectos en las mujeres de la cohorte más joven, nacidas entre 1971-1975; esta situación deriva del incremento del grupo que no experimentó ninguna transición, así como del inicio de la vida reproductiva a partir de la primera relación sexual. No obstante lo anterior, cuatro de cada diez mujeres jóvenes continuaron apegándose al patrón normativo.

Para determinar la existencia de calendarios familiares diferentes según las trayectorias migratorias de las tres cohortes de hombres y mujeres de la Eder, Sebille (2005) busca averiguar qué tanto la primonupcialidad y la primofecundidad fueron afectadas por la existencia o no de la migración. Para ello construye ocho trayectorias migratorias. Sus resultados revelan que los hombres que al migrar se encontraban solteros, independientemente de que su lugar de destino fuera rural o urbano, tardaron más tiempo que los sedentarios en casarse, lo cual evidencia que los proyectos migratorios y el tiempo dedicado a la mi- 
gración sí parecen influir en el calendario de la nupcialidad. En contraste, las mujeres no migrantes fueron las que mayormente comenzaron a diferir su primera unión en las generaciones más jóvenes. A pesar de este aplazamiento en la nupcialidad, el autor determina que la migración no afectó el inicio de la vida reproductiva una vez casados.

Tuirán (2001), centrándose en el examen de la transición de la adolescencia a la edad adulta de las mujeres, emplea la Encuesta sobre Determinantes de la Práctica Anticonceptiva en México (Edepam, 1988), misma que contiene información sobre la salida de la escuela, el primer trabajo, la primera salida del hogar, la primera unión conyugal y el primer hijo. Mediante la distribución porcentual de cinco cohortes de mujeres alguna vez unidas, establece cuántas han experimentado determinado número de transiciones. Presenta la información desagregada por localidades: rurales, semiurbanas y urbanas. El autor evalúa la hipótesis de la trayectoria típica o normativa de estas transiciones y advierte que sólo una proporción reducida de cada cohorte sigue esta trayectoria, a pesar de registrarse una mayor prevalencia de todas y cada una de las transiciones que dan forma al paso de la adolescencia a la edad adulta.

Finalmente, el trabajo de Coubès y Zenteno (2005) es el antecedente más directo de la investigación que aquí se realiza, ya que presenta un estudio sobre la salida de la escuela, el primer empleo y la primera unión conyugal, tomando como base la muestra rural y urbana de la Eder 1998; su objetivo es determinar si en el país ha prevalecido el modelo normativo. Los autores parten del supuesto de que con el proceso de modernización de las sociedades occidentales, en conjunto con las transformaciones experimentadas en el interior de la familia, se ha generado una estandarización de las etapas biográficas con la existencia de límites formales de edades, lo cual ha producido una regularidad en los patrones y en los tiempos en que las personas realizan ciertas transiciones que las encaminan hacia la adultez en un orden socialmente esperado y regulado por instituciones sociales tales como la escuela, el trabajo y la familia.

En el caso de estas transiciones el orden normativo estaría establecido por la salida de la escuela, el acceso al primer empleo y la entrada en unión conyugal. Coubès y Zenteno parten de considerar que el modelo normativo especifica una edad apropiada para las transiciones importantes de la vida, con normas que marcan un calendario individual adecuado para éstas; sin embargo reconocen que para países en desarrollo como México no se puede hablar de un modelo de este tipo 
en el paso a la adultez, ya que el Estado, la comunidad y la familia operan de forma muy diferente a la de los países desarrollados. En los primeros los lazos familiares son fuertes y juegan un papel fundamental en la socialización de los individuos, en tanto que la comunidad es una instancia de mediación importante, sobre todo para el caso de las áreas rurales o indígenas, mientras que las instituciones del Estado se muestran cada vez más deficientes, por lo que poco garantizan la existencia de una vida digna para su población. Contrario a ello, en Estados Unidos y en algunos países europeos los lazos familiares son débiles y se da un mayor énfasis a la autonomía de la persona, tanto en el ámbito financiero como en el residencial (Coubès y Zenteno, 2005).

Una de las constantes que atraviesan los trabajos anteriores es la presencia de la transición a la vida conyugal heterosexual, la cual parece ser un parteaguas indiscutible que diferencia la vida de las personas al facilitar el surgimiento de otro tipo de transiciones y actividades, tal como las relaciones sexuales, el uso de métodos anticonceptivos, la reproducción y la crianza de los hijos, a la vez que entra en conflicto con ámbitos tales como la escuela, el empleo y la migración. Los cuestionamientos que plantean estos trabajos llevan a sus autores a explorar las diferencias por sexo y cohorte, y en ocasiones por lugar de residencia, en la conformación de estas trayectorias. Entre sus objetivos destaca el precisar aquellas secuencias más comunes que se conforman a partir de las trayectorias enunciadas, y en ocasiones probar la prevalencia del modelo normativo dentro de la sociedad mexicana.

Sin embargo pocas veces se plantea indagar a fondo la relación que el género, el nivel socioeconómico y la cohorte de nacimiento guardan en la conformación de estas trayectorias, así como precisar cómo es que estas diferencias se interrelacionan para producir secuencias con determinados órdenes y temporalidades. Este trabajo apuesta por esbozar trayectorias que por sí mismas hablen de espacios y tiempos marcados por las desigualdades, mismas que más que abatirse parecieran afianzarse con el transcurrir del tiempo. Dadas estas desigualdades, la convergencia hacia un modelo único entre hombres y mujeres, así como entre niveles socioeconómicos, es prácticamente nula, a pesar de la reducción de la brecha educativa por género y de la mayor participación femenina en el mercado laboral, ya que éstos son los indicadores más visibles que encubren la presencia de otras disparidades más arraigadas y resistentes al cambio, como pudiera ser la calidad en la educación por nivel de ingreso y localidad, los mercados laborales segmentados en función del sexo, el pago diferencial que hombres y 
mujeres reciben por trabajos iguales, y las relaciones desiguales que éstos establecen entre sí dentro de las relaciones de pareja, entre otros aspectos.

\section{Datos y métodos}

La base de datos empleada es la submuestra urbana de la Encuesta Demográfica Retrospectiva (Eder, 1998). Ésta es la primera encuesta mexicana que recolectó historias de vida de una muestra representativa de hombres y mujeres residentes en todo el territorio nacional (Coubès, Zavala de Cosío y Zenteno, 2005). Los datos contienen la historia individual de las cohortes de mexicanos nacidas en 1936-1938 (cohorte antigua), 1951-1953 (intermedia) y 1966-1968 (joven) y que sobrevivían en el momento en que se practicó la encuesta.

Dicha muestra es representativa en el ámbito nacional para ambos sexos, tres cohortes y dos tamaños de localidad (menos de 15000 habitantes y 15000 y más). Estos últimos son mencionados en la fuente de datos como "rural" y "urbano" (Coubès, Zavala de Cosío y Zenteno, 2005). Esta investigación se enfocó exclusivamente en la población urbana, ${ }^{7}$ definida a partir de la residencia en el momento de la encuesta. Para la parte descriptiva se analizó la información sin ponderar ${ }^{8}$ de 1266 personas, $54.3 \%$ mujeres y $45.7 \%$ hombres, mientras que para los modelos de historia de eventos se tomó la información de éstos en años-persona, lo que permitió contar con un número bastante aceptable de observaciones.

En la parte descriptiva se presentan los porcentajes de las trayectorias que se obtuvieron de la combinación de las tres transiciones, diferenciando a su vez las secuencias en donde las personas no asistie-

\footnotetext{
${ }^{7}$ Este artículo forma parte de una investigación más amplia que analizó las transiciones y trayectorias de hombres y mujeres conforme a tres ejes de diferenciación: cohorte de nacimiento, género y nivel socioeconómico. Dada la complejidad de manejar estas tres categorías y los subgrupos que de ellas provenían, se optó por no incluir una cuarta categoría de diferenciación: la localidad rural-urbana. Sin embargo, en los modelos de historia de eventos se incluye esta variable para determinar la importancia del lugar de socialización cuando las transiciones analizadas comenzaban a suceder.

${ }^{8}$ En el uso de ponderadores Coubès, Zavala de Cosío y Zenteno (2005: 23) advierten de la dificultad de extender el carácter representativo de la población en el momento de la encuesta hacia el pasado. La muestra de la Eder es representativa de la población sobreviviente de tres cohortes en 1998, pero la representatividad de la muestra es menos precisa a mayor incursión en los años anteriores. Para que la muestra fuera representativa a cada año-calendario de la historia de vida de las tres cohortes se necesitarían factores de ponderación que variaran a través del tiempo.
} 
ron a la escuela. Para permitir la comparabilidad entre cohortes se cuidó que las tres transiciones hubieran sucedido antes de los 30 años. La base de datos de la Eder no permite distinguir el orden de dos transiciones cuando ambas se presentan en el mismo año, por ello se adoptó el supuesto del ordenamiento temporal normativo cuando así sucedió. ${ }^{9}$ Para profundizar en el conocimiento de las trayectorias más representativas de cada género, se seleccionaron dos para hombres y dos para mujeres y se calcularon mediante tablas de vida las edades medianas de las transiciones que conformaron estas secuencias, así como los intervalos promedio entre la primera y la segunda, y entre la segunda y la tercera transición, cuando hubo una tercera, además del número de años transcurridos entre el inicio y el final de la secuencia para determinar su duración. La intención de este ejercicio fue delinear algunas expresiones normativas de género que marcan y regulan los tiempos y los ritmos de tránsito a la adultez.

Para evitar caer en la falacia de secuencia ${ }^{10}$ este cálculo se hizo tomando solamente al subgrupo de población de cada cohorte que siguió las transiciones en el orden marcado por las secuencias elegidas, excluyendo a todos los demás. Para distinguir principalmente la influencia que el nivel socioeconómico tuvo en el inicio de estas trayectorias se ajustaron dos modelos de historia de eventos.

\section{Una aproximación a las trayectorias}

En los siguientes apartados se presenta un análisis de las trayectorias más comunes a las tres cohortes de este estudio. En total son 15 las secuencias que se pueden construir a partir de las tres transiciones enunciadas, las cuales son identificadas con un número para facilitar su manejo. Las primeras cinco inician con la salida de la escuela, las secuencias 6 a 8 comienzan con el logro del primer trabajo estable, las siguientes tres con la unión conyugal, y finalmente las secuencias

${ }^{9}$ Fue bajo el porcentaje en donde ocurrieron situaciones de simultaneidad, presentándose el mayor número de casos entre la salida de la escuela y el primer trabajo estable, el cual varió entre el 9 y el 16\%, no observándose una tendencia definida por sexo o cohorte.

${ }^{10}$ Esta falacia se presenta cuando, usando medidas de tendencia central, se pretende mostrar una imagen sobre la secuencia estándar en el curso de la vida construida a partir de ciertas transiciones, y se le atribuye a toda la población de determinada cohorte, lo cual no es lo más adecuado debido a que no todas las personas experimentan dichas transiciones (Billari, 2001: 123). 


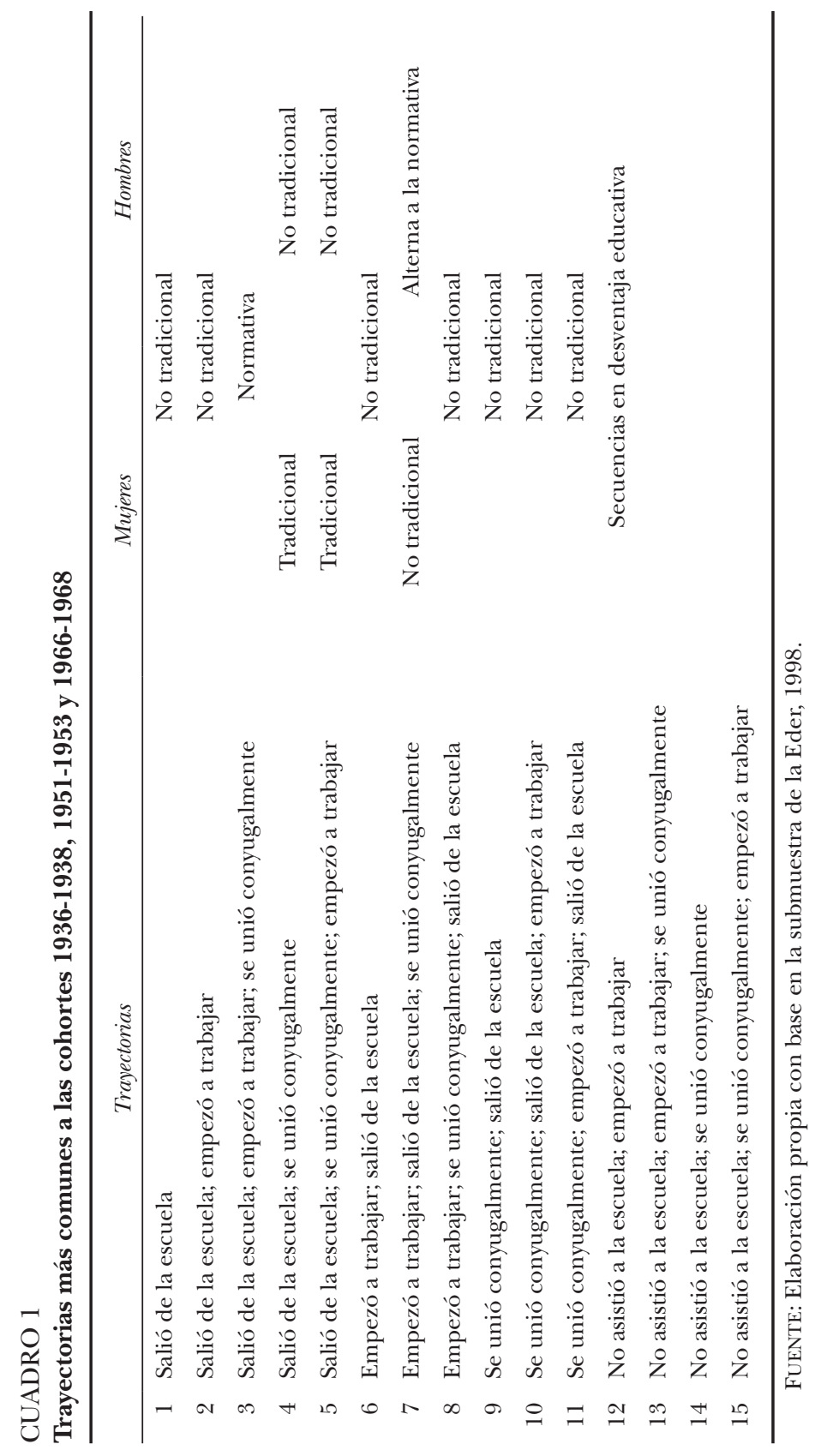


12 a 15 agrupan a personas que no asistieron a la escuela, por lo que no tuvieron la posibilidad de salir del sistema educativo, situación que se consideró necesario distinguir del resto, ya que de entrada tuvieron desventajas que por fuerza influyeron en sus otras transiciones.

El curso de vida hace referencia al modelo normativo, el cual puede ser definido como aquel que es experimentado por la mayoría de la población y que además, conforme a la expectativa social, ha de ser experimentado en cierto momento de la vida (Elder, 1978). En el esquema que se presenta, la secuencia normativa sería la número 3. Cabe mencionar que esta secuencia es más propia en los países desarrollados, en donde la brecha de género en los distintos ámbitos de la vida social es reducida, por lo que se espera que tanto los hombres como las mujeres ingresen al sistema escolar, concluyan estudios avanzados, desempeñen su profesión y, luego de haber acumulado algún capital humano y económico, inicien su vida en pareja.

$\mathrm{Al}$ respecto Coubès y Zenteno (2005), a partir de estas mismas tres transiciones, encuentran que si bien el modelo normativo ha sido relativamente importante en México, especialmente para los varones, no se ha constituido como un patrón único. Asimismo afirman que en el país no existe una convergencia hacia un modelo particular, sobre todo en el caso de las mujeres, pues coexisten secuencias de vida tradicionales asociadas a la división sexual del trabajo, con maneras más diversas de conformar la vida adulta. Finalmente, previenen sobre las limitaciones de emplear el modelo normativo en un contexto de creciente heterogeneidad social y educativa como es el mexicano, ya que en el país un amplio porcentaje de la población abandona a edades precoces la escuela, por lo que si esto ocurre las posibilidades de iniciar un empleo o de unirse posteriormente son amplias, y la secuencia normativa se vuelve inevitable.

La diversidad educativa y laboral, aunada a las diferencias entre géneros, niveles socioeconómicos, contextos de residencia y cohortes, lleva a vislumbrar en México cierta expectativa social en el orden en que se esperaría que cada persona realice estas transiciones, produciendo trayectorias que pueden parecerse al modelo normativo, pero con significados sociales distintos, o pueden incluso crearse trayectorias alternativas adaptadas a cada contexto. Lo anterior en total concordancia con el principio del tiempo y el lugar, el cual afirma que el curso de vida individual se encuentra imbricado y formado tanto por el tiempo histórico, como por el lugar en que las experiencias de vida de las personas se realizan (Elder, Kirkpatrick y Crosnoe, 2003). 
Así se podría esperar que los varones sigan el llamado modelo normativo en mayor medida que las mujeres, o en todo caso alteren el orden de la primera transición, comenzando por el ingreso al mercado de trabajo para después dejar la escuela, pues se sabe que en momentos de necesidad económica las familias, sobre todo las más pobres o las de las áreas rurales, tienden a mandar a sus jóvenes, principalmente varones, al mercado de trabajo para obtener ingresos adicionales (Giorguli, 2002), debiendo éstos combinar por un tiempo las dos actividades. En ambos casos el inicio de la vida matrimonial sería la última transición.

En tanto en las mujeres, sobre todo en aquellas ubicadas en ámbitos menos escolarizados y con mayores carencias económicas, es posible esperar que el orden de las transiciones sea salir de la escuela, casarse y comenzar su procreación, con escasas e intermitentes participaciones dentro del mercado laboral (Ariza, 2000: 194). En estos espacios la temprana deserción escolar femenina es resultado del aumento de su participación en las tareas del hogar y en el cuidado de los menores para apoyar a las mujeres adultas que han ido insertándose cada vez más en la fuerza de trabajo y requieren del apoyo de los demás miembros de la familia para compatibilizar los roles domésticos con los laborales (Rendón, 2004; Giorguli, 2006). Este ordenamiento estaría respondiendo a la manera tradicional de constituir el género, la familia, la sexualidad y la división sexual del trabajo.

En este artículo se entiende por tradicional al conjunto de patrones culturales de una o varias generaciones heredado de las anteriores y trasmitido a las siguientes (Giddens, 1999). Giddens considera que las características distintivas de la tradición son el ritual y la repetición realizados dentro de grupos, comunidades o colectividades, ya que las tradiciones no pertenecen al comportamiento individual, sino que requieren de su reiteración colectiva. Lo que es distintivo acerca de una tradición es que define un tipo de verdad. Quien sigue una práctica tradicional no se hace preguntas acerca de las alternativas, en todo caso realiza ciertos cambios o adaptaciones en las etapas más tardías de una transición, pero sin alterar radicalmente el contenido o significado de dicha práctica, que permanece en gran medida incuestionable. Ello no significa que la persona no goce de una libertad de elección y acción, sino que ésta se encuentra circunscrita a sus condiciones particulares de vida, desarrollada dentro de oportunidades y circunstancias históricas y sociales constreñidas. 
Con esa idea en mente se clasificaron las secuencias en tradicionales y no tradicionales (cuadro 1). Con estos términos nos referimos a aquellas secuencias esperadas y sancionadas por la sociedad y la cultura en que hombres y mujeres se desarrollan, o que por el contrario, se excluyen de la aspiración del subgrupo de pertenencia. Las secuencias tradicionales fueron la 4 y 5 para el caso de las mujeres, ya que éstas estarían iniciando su unión conyugal una vez fuera de la escuela, con posibilidad o no de integrarse al mercado laboral estable luego del matrimonio.

En contraste, y justamente por el orden en que está colocado el primer trabajo o la ausencia del mismo, para los varones estas dos secuencias se consideran no tradicionales. En el mismo sentido se encuentran los trayectos $1,2,6,8,9,10$ y 11 , catalogados como no tradicionales tanto para hombres como para mujeres, dado que contienen sólo una o dos transiciones, o se presentan al inicio de la unión conyugal antes de la integración a la fuerza de trabajo o de la salida de la escuela, lo cual no resultó muy común al revisar su distribución porcentual.

La secuencia 3 se mantuvo con el nombre de normativa y la 7 se enunció como no tradicional para el caso de las mujeres, ya que no era común, sobre todo en las cohortes antiguas, que iniciaran su transición hacia la adultez con el trabajo y posteriormente dejaran la escuela. En cambio en los hombres ésta es una trayectoria alternativa, presente en casi una cuarta parte de ellos. Mención aparte merecieron las trayectorias 12 a 15, mismas que al no incluir el ingreso al sistema educativo indicaron el comienzo de una serie de desventajas sociales acumuladas.

\section{Trayectorias de mujeres y hombres}

Casi tres de cada cuatro mujeres, sobre todo de las cohortes más jóvenes, tuvieron como transición de inicio la salida de la escuela (véase el cuadro 2 para todo este apartado). ${ }^{11}$ Esto indica la importancia de la escolaridad en la vida de las mujeres mexicanas y la presencia de la unión conyugal y del primer trabajo estable luego de haber terminado o abandonado sus estudios.

\footnotetext{
${ }^{11}$ Para facilitar al lector el manejo de la información, el cuadro 2 agrupa en una sola las secuencias 12 y 15 , que no incluyeron la escolaridad por tener pocos casos; lo mismo sucedió con aquellas no tradicionales, tal como la 1, 6, 8, 9, 10 y 11, que sólo contenían una transición, o iniciaban con el matrimonio.
} 


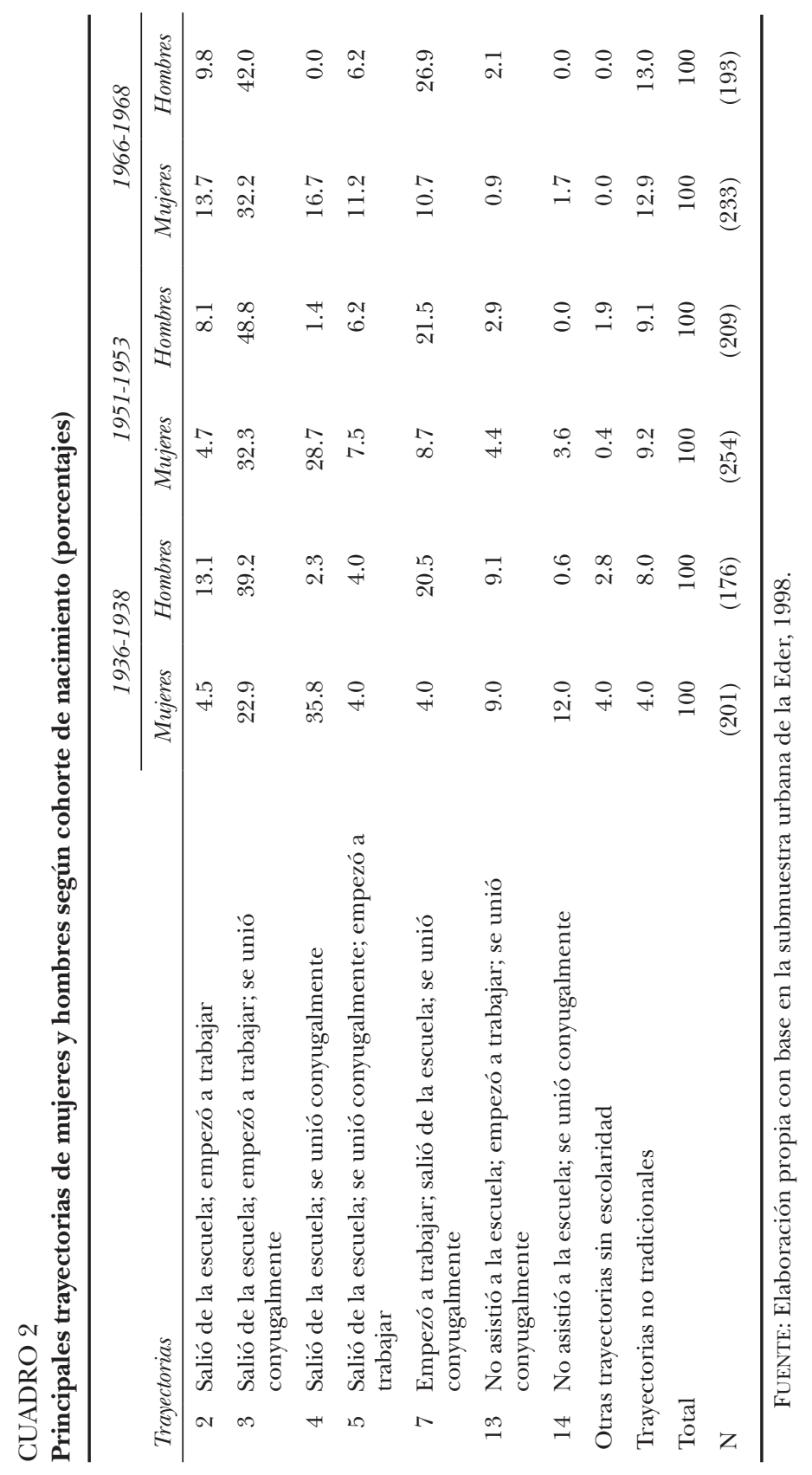


Entre la población femenina destacaron la trayectoria 3, llamada normativa, y la 4, tradicional; ambas comienzan con la salida de la escuela, pero en la 3 las mujeres entraron al mercado laboral y al final se casaron, mientras que en la 4 hasta los 29 años no se había presentado una entrada al mercado de trabajo, aunque es posible que algunas de estas mujeres realizaran trabajo extradoméstico después de esa edad.

El ingreso a la vida laboral, como primera transición, agrupó a $18 \%$ de ellas; en la cohorte antigua este porcentaje se debió a la ausencia de escolaridad, en tanto que en las cohortes jóvenes la trayectoria 7, que combina escuela y trabajo, comenzó a destacar. Iniciar su adultez con la unión conyugal fue una situación poco común, más bien presente entre las mujeres de la cohorte antigua que no asistieron a la escuela, las cuales a su vez siguieron las secuencias 13 y 14 .

En la población masculina la salida de la escuela como transición de inicio agrupó a casi seis de cada diez hombres de las distintas cohortes, en tanto que el comenzar su trayecto trabajando fue experimentado por los restantes cuatro de cada diez, lo cual es congruente con la división tradicional del trabajo que marca para ellos una edad de inicio laboral más temprana y una actividad extradoméstica más intensiva que la de las mujeres. En contraste, fue prácticamente nulo el porcentaje de hombres que primero se casó.

En ellos sobresalen las secuencias 3, normativa, y la 7, misma que pudiera ser considerada como una alternativa en el tránsito a la adultez masculina mexicana. La trayectoria 3 fue común para hombres y mujeres, pero destaca su predominio en los varones. Por su parte la secuencia 7, también común a ambos a partir de la cohorte intermedia, fue aumentando su porcentaje conforme el tiempo transcurrió, aunque su presencia siempre fue mayor entre la población masculina (véase la gráfica 1).

Las secuencias no tradicionales relacionadas con la ausencia de trabajo (1, 4, 9 y 14) no fueron opciones para la población masculina, ya que laborar antes de los 30 años es una transición que prácticamente todos realizaron. Ello confirma que el trabajo es una actividad indispensable para darle sentido y dirección a la vida de los hombres, pues contribuye a formar su identidad de género dentro de una sociedad en donde el sexo biológico pareciera ligarlos ineludiblemente al ámbito del espacio público. Sin embargo la secuencia 12, que indica que la persona sólo realizó la transición al primer trabajo, tampoco fue común en los varones, situación que revela que si bien contar con un trabajo estable parece ser sumamente importante para ellos, esto 


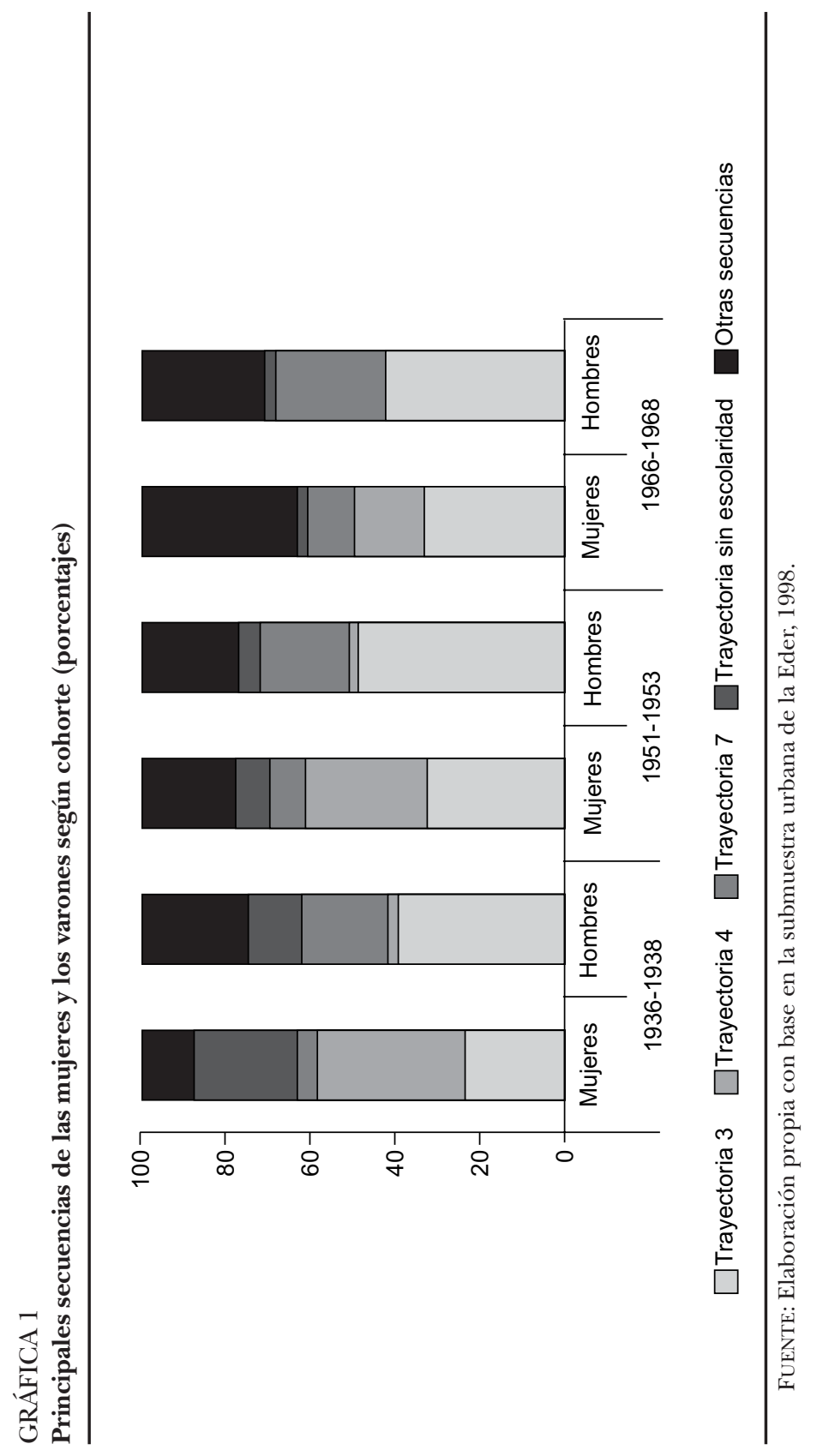


se complementa con las otras dos transiciones en su configuración como adultos.

Las secuencias sin escolaridad (12 a 15) disminuyeron drásticamente a partir de la segunda cohorte, aunque siempre estuvieron más presentes entre las mujeres que entre los hombres. Destaca la amplitud de trayectos presentes en la vida de estas personas, en las cohortes más antiguas como producto de la ausencia de escolaridad, sobre todo entre la población femenina, y como resultado de la realización de trabajo extradoméstico, mientras que en la cohorte joven esta variedad se atribuyó a la posposición de la nupcialidad hasta después de los 30 años de edad, y en las mujeres, además, a su incorporación plena al ámbito educativo y al mercado laboral (véase en la gráfica 1 las secuencias sin escolaridad y otras trayectorias).

En los hombres las secuencias 3 y 7 parecieron primar a lo largo del tiempo, mientras que en las mujeres la 3 y la 4 coexistieron con otras, como las relacionadas con la falta de educación formal (13 y 14) y con el inicio de la adultez a través del primer empleo, como la trayectoria 7 . Se advierte cierta estabilidad en la conformación de la adultez en los varones y una mayor variedad de trayectorias en las mujeres, sobre todo en la cohorte joven, lo que pudiera mostrar mandatos de género más rígidos para ellos porque no pueden posponer el inicio de su vida productiva, y un tanto más flexibles para ellas en el tránsito a la adultez.

\section{Las trayectorias de la cohorte antigua: 1936-1938}

La forma en que las personas conciben el mundo social depende en gran parte de sus condicionantes sociohistóricas, marcadas por el tiempo en que nacieron y vivieron sus primeros años. Una forma de aproximarse al estudio de este cambio es mediante las cohortes de nacimiento, cuyos miembros enfrentan una misma historia social, con eventos, oportunidades y límites dados por la sociedad en un momento determinado (Alwin y McCammon, 2003).

En el momento en que los miembros de la cohorte antigua se encontraban en la infancia, en la década de los cuarenta, el país iniciaba un proceso de industrialización y urbanización y comenzaba a prosperar económicamente, sin embargo dentro del campo mexicano la población vivía aislada y en pobreza, y en torno a las ciudades se extendían cinturones de miseria conformados por personas que mi- 
graban del campo a la ciudad en busca de oportunidades (Alba y Potter, 1986). Como consecuencia de la urbanización y de la industrialización, la población económicamente activa, mayoritariamente masculina, crecía en forma acelerada (Parrado y Zenteno, 2005).

A causa de los mandatos de género la población femenina vivía bajo una fuerte dependencia y sumisión a la autoridad masculina. La mayoría de las mujeres se dedicaba de manera exclusiva al trabajo doméstico, y una vez casadas al cuidado de los hijos; ellas se hallaban insertas en un contexto donde predominaba la nupcialidad precoz, con una edad promedio a la primera unión de 20.7 años para las mujeres y de 23.9 años para los hombres; la fecundidad era intensiva y el uso de métodos anticonceptivos nulo (Rendón, 2004; Brugeilles y Samuel, 2005).

Las oportunidades ocupacionales para ellas estaban muy limitadas y mayormente dirigidas a las solteras. Es por esto que apenas la mitad de las mujeres de la cohorte antigua, quienes llegaron a su vida productiva a mediados de la década de los cincuenta, realizó trabajo extradoméstico antes de los 30 años.

La escolaridad aún no se encontraba establecida como una opción de desarrollo; había pocos profesores y escasas escuelas, la mayoría concentradas en las localidades urbanas, y la educación formal era un privilegio asignado mayoritariamente a los varones, sobre todo urbanos. Para 1940 el porcentaje de personas consideradas como analfabetas era de $54 \%$ y el número medio de años aprobados en la escuela de la población mayor de 15 años era de apenas dos (Greaves, 2011).

Este panorama sociohistórico se ve reflejado fielmente en las trayectorias de la cohorte antigua. En las mujeres destacó en primer lugar la secuencia 4 o tradicional (36\%) (cuadro 2), donde éstas salieron de la escuela y posteriormente se casaron, lo que manifiesta su ausencia de ingreso al mercado laboral, en total concordancia con su rol tradicional de madres, esposas y amas de casa.

También se presentó la secuencia 3 (23\%), seguida por quienes pudieron ingresar al mercado laboral, aunque en menor medida que la 4. Otras secuencias presentes fueron aquellas donde las mujeres no contaron con algún grado de educación formal, mismas que agruparon a una cuarta parte de la población femenina de esta cohorte; de ellas destacaron la 13 y la 14 (9 y 12\% respectivamente), caracterizadas sólo por el trabajo y/o la unión conyugal.

La contraparte masculina siguió mayoritariamente las secuencias 3, 7, 2 y 13 (39, 20.5, 13 y 9\% respectivamente). Las dos primeras fueron una constante que acompañó el tránsito a la adultez de los 
varones mexicanos, la tercera agrupó a quienes hasta los 29 años no habían comenzado una relación conyugal, en tanto que la última fue una secuencia esperada para ellos en un tiempo en que la educación formal no era universal, ya que en ésta la escolaridad estuvo ausente.

\section{Las trayectorias de la cohorte intermedia: 1951-1953}

A comienzos de 1960 la cohorte antigua contaba ya con más de 23 años y la intermedia, nacida entre 1951 y 1953, vivía sus primeros años en un periodo de rápido crecimiento económico en México, aun bajo el modelo industrial de sustitución de importaciones presente desde 1940. No obstante, a principios de la década de los setenta comenzaron a presentarse las primeras señales de agotamiento de este modelo económico (Alba y Potter, 1986).

Las condiciones laborales de los trabajadores empezaron a deteriorarse, se estancó el proceso de asalarización de la fuerza de trabajo y se presentó el fenómeno de la terciarización del empleo (García, 1988; Rendón, 2003), mismo que abrió paso a la incorporación paulatina de las mujeres al mercado de trabajo y a la feminización laboral (Oliveira, 1990; Oliveira, Ariza y Eternod, 2001; Rendón, 2004).

En 1960 el número medio de años aprobados en la escuela de la población en general seguía siendo de tan sólo 2.2 años y el analfabetismo se encontraba presente en cuatro de cada diez personas mayores de 15 años (Muñoz y Suárez, 1994; Alba, 1989). Las uniones conyugales de las mujeres continuaban siendo tempranas, los nacimientos de los primeros hijos no se retrasaban y la esterilización femenina comenzaba a ser el método anticonceptivo al que mayormente se recurría después de alcanzar el tamaño de familia deseado (Zavala de Cosío, 1996).

En las mujeres de la cohorte intermedia la secuencia 3 ganó importancia (32\%), desplazando a la 4 o tradicional (29\%); en ambas la transición de inicio fue la salida de la escuela (cuadro 2). El inicio de la adultez a través de la salida de la escuela es un reflejo del aumento de la escolaridad en esta cohorte, puesto que aproximadamente $91 \%$ de las mujeres y $96 \%$ de los hombres asistieron al menos un año a la escuela. El hecho de que ganara en porcentaje la trayectoria 3 , en donde la incorporación al mercado laboral fue la segunda transición, es indicativo del valor que comenzó a adquirir el trabajo extradoméstico en algunas mujeres de esta generación como forma de 
iniciar la adultez, aun cuando también pudo ser un evento forzado ante las carencias del hogar de origen. Esta misma situación repercutió en que apareciera una tercera trayectoria para ellas, la 7 , no tradicional (9 por ciento).

En los varones la trayectoria 3 también concentró el mayor porcentaje $(49 \%)$, seguida de la $7(21.5 \%)$. Otras como la secuencia 2, en donde el matrimonio no había sucedido hasta antes de los 30 años, continuaron vigentes $(8 \%)$. En contrapartida, las secuencias donde las personas no tuvieron la oportunidad de estudiar (12 a 15) estuvieron presentes en $8 \%$ de la población femenina y en $5 \%$ de la masculina de esta cohorte, disminuyendo sustancialmente su participación con relación a la cohorte antigua. Esta reducción del porcentaje de personas sin escolaridad pudiera ser un reflejo de los resultados obtenidos al haberse aplicado en el país a partir de 1960 el Plan de Once Años, el cual permitió a más niños acceder a la educación primaria tanto en el campo como en la ciudad, sobre todo en los primeros tres grados de escolaridad, y contar por primera vez con libros de texto gratuitos (Muñoz y Suárez, 1994; Greaves, 2011).

Así, la disminución de las trayectorias sin escolaridad y el consiguiente aumento de las trayectorias educativas son un reflejo de la intervención del Estado, que mediante una política educativa buscó abatir las desigualdades en el plano educativo elemental. Sin embargo, el hecho de que aun en esta cohorte las mujeres concentraran un mayor porcentaje de personas sin educación formal es una clara expresión de la preferencia de las familias por invertir en mayor medida en el capital humano de los varones, pues lo consideraban un bien necesario para su futuro familiar (Giorguli, 2002).

\section{Las trayectorias de la cohorte joven: 1966-1968}

La cohorte joven vivió en un país mayoritariamente urbano en el que la escolarización de los niños y la inserción de las mujeres en el mercado de trabajo asalariado comenzaron a progresar. Sin embargo, también creció en un contexto de depresión económica constante que comenzó en la década de los setenta y que tuvo sus mayores expresiones en 1981 y en 1994, cruzando permanentemente todos los ámbitos de su vida (Samuel y Sebille, 2005; Brugeilles y Samuel, 2005).

México había continuado expandiendo los servicios educativos, mayormente en el nivel de primaria, lo que llevó a que un mayor nú- 
mero de personas tuvieran acceso a la escuela. En 1970 la población alcanzó una escolaridad promedio de 3.4 años y el analfabetismo se situó en 31.6\% entre las personas de 15 años y más (Alba, 1989; Muñoz y Suárez, 1994).

Sin embargo, a inicios de los ochenta, justo cuando la cohorte joven se encontraba entre los 12 y los 14 años, el país había comenzado a realizar su transición hacia el modelo industrial exportador y de apertura económica liberal. Esta etapa se caracterizó por el continuo deterioro de las condiciones laborales y de los ingresos en México, asî como por la reducción del empleo asalariado (Parrado y Zenteno, 2005). La terciarización del empleo no asalariado creció, así como el trabajo familiar sin pago y el asalariado a destajo, además aumentó la población trabajadora sin acceso a prestaciones (Rendón, 2003).

Algunos sectores de mujeres que tradicionalmente se habían mantenido al margen del mercado laboral, tales como las de mayor edad, las casadas o unidas, las que tenían hijos en edad escolar y las de más baja escolaridad comenzaron a participar en la actividad económica (García y Oliveira, 1991). Este cambio en las tendencias tradicionales de retiro de las actividades económicas extradomésticas después del matrimonio pudo deberse a la necesidad de las familias de contar con un mayor ingreso corriente, pero también al descenso de la fecundidad, a las transformaciones experimentadas en las relaciones de género, al aumento en las tasas de la migración masculina, a la elevación de los niveles educativos de las mujeres y a la frecuencia de los divorcios y las separaciones conyugales (García y Pacheco, 2000; Rendón, 2003 y 2004).

Otra esfera que sufrió transformaciones, aunque más lentas, fue la vida reproductiva, marcada sobre todo por la postergación de la edad de las mujeres a la primera unión y a la vida fecunda, así como por una anticoncepción cada vez más frecuente durante las uniones (Brugeilles y Samuel, 2005).

En este contexto, la trayectoria 3 siguió presentándose en una de cada tres mujeres de la cohorte joven (cuadro 2), aun cuando no llegó a establecerse como el patrón dominante ya que las secuencias 4, 2 y 5 , en ese orden, también fueron seguidas por estas mujeres, teniendo todas en común la salida escolar como primera transición, lo que a su vez muestra que el ingreso al sistema educativo fue prácticamente universal a partir de la década de los setenta, ya que en esta cohorte el 98\% de las mujeres y de los hombres asistió a la escuela, aunque su edad mediana de egreso, si bien fue mayor que la de las otras dos cohortes, sólo se ubicó en los 15.7 y 16.7 años respectivamente. Esta 
temprana edad pudiera explicarse, al menos en parte, por el deterioro de la estabilidad económica del país, situación que pudo haberles impedido un aumento todavía mayor en las edades medianas de salida de la escuela.

Asimismo, estas mujeres aumentaron masivamente su participación laboral antes de los 30 , con un $78.5 \%$, por lo que las secuencias no tradicionales 7, 6 y 8 , relacionadas con el ingreso a la vida productiva como transición de inicio, cobraron importancia (18 por ciento).

En los hombres de esta cohorte las secuencias 3 y 7 continuaron primando, incluso esta última aumentó al pasar de 20.5 a $27 \%$ entre las cohortes extremas, posiblemente al buscar apoyar a la economía familiar dentro de un contexto de crisis económica nacional mientras estudiaban. La postergación de la unión conyugal hasta después de los 30 años estuvo presente en el 18\% de ellos, tal como lo indican las trayectorias 2 y 6 .

\section{La temporalidad de las secuencias de la población femenina y masculina}

En esta sección se profundiza en la temporalidad: la duración de los intervalos entre transiciones y la duración total entre el inicio y el final de las trayectorias más comunes para las mujeres (3 y 4 ) y los hombres (3 y 7). ${ }^{12}$ Esta información se presenta en gráficas con la intención de ofrecer un panorama longitudinal de una parte del curso de vida y facilitar la observación y la comparación de experiencias diferenciadas en el tránsito hacia la adultez, producto en gran parte de normativas de género.

\section{La trayectoria 3 o normativa: divergencias por sexo}

La edad mediana de la salida escolar de las mujeres que siguieron la secuencia 3 se situó entre los 13 y los 15.7 años en las cohortes extremas (gráfica 2), aumentando la escolaridad con el transcurso del tiempo gracias a los logros en la cobertura escolar que se alcanzaron en el país durante la segunda parte del siglo Xx (Castro y Gandini, 2006). Si se comparan estas edades con las medianas del total de la población fe-

\footnotetext{
${ }^{12}$ Se cuidó que las trayectorias elegidas tuvieran más de 35 casos en cada cohorte.
} 


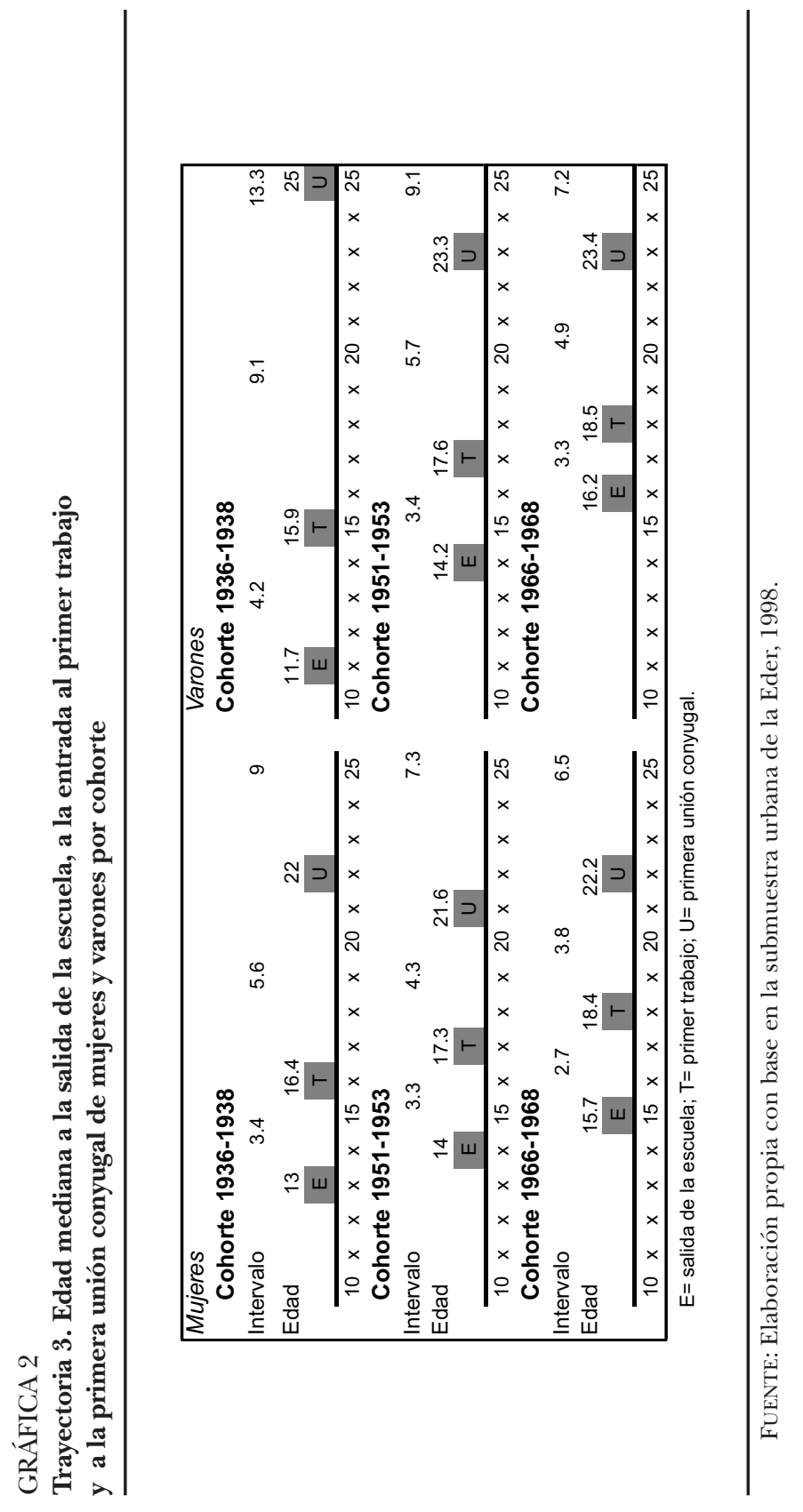


menina en cada cohorte, ${ }^{13}$ se advierte que las mujeres de las dos cohortes más antiguas que siguieron esta secuencia estuvieron casi un año más en la escuela que el resto de sus compañeras de cohorte, en tanto que en la cohorte joven esta edad fue idéntica a la del total de la población. Las mujeres que siguieron este trayecto pudieron haber acumulado un capital humano mayor o similar al del resto de sus compañeras de cohorte. ${ }^{14}$

Una vez fuera del sistema escolar estas jóvenes dejaron transcurrir aproximadamente tres años antes de desempeñar un trabajo extradoméstico que durara al menos un año, y en el inter pudieron haberse dedicado a realizar labores domésticas o quizá trabajos fuera del hogar de manera intermitente. Es interesante destacar que su edad mediana de entrada al mercado laboral fue mucho menor que la del total de las mujeres de cada cohorte, quizá como efecto de su escolaridad, que si bien no era muy elevada, pudo haber sido un factor que promoviera su inserción en el área laboral.

En contraste con el aumento cada vez mayor, y estadísticamente significativo, ${ }^{15}$ de las edades medianas de salida de la escuela y del primer trabajo de las mujeres, la edad mediana a la primera unión conyugal se mantuvo prácticamente estable en el tiempo, situándose cerca de los 22 años. Esta edad a la unión fue mayor a la experimentada por el resto de sus compañeras de cohorte. ${ }^{16}$ Entre el inicio y la conclusión de la trayectoria transcurrieron 9 años en la cohorte antigua

${ }^{13}$ Las edades medianas de salida de la escuela para el total de la población fueron $12,13.1$ y 15.7 años para las cohortes antigua, intermedia y joven, respectivamente.

${ }^{14}$ La encuesta no permite identificar qué tanto las edades de salida reflejan el grado escolar al que las personas llegaron, pero la variable de nivel educativo indica la existencia de cierta correspondencia entre estas edades y el logro escolar.

${ }^{15}$ La prueba de regresión de Cox para la igualdad de curvas de supervivencia de la edad mediana de salida de la escuela muestra diferencias estadísticamente significativas según cohorte de mujeres. El estadístico Wald $\chi^{2}$ es de $(1)=9.16$ con $\operatorname{Pr}>\chi^{2}=0.0103$. Por su parte la prueba de Wilcoxon (Breslow) para la igualdad de funciones de supervivencia de la edad mediana al primer empleo muestra diferencias estadísticamente significativas por cohorte de mujeres. El estadístico $\chi^{2}$ es de $(1)=8.08$ con $\operatorname{Pr}>\chi^{2}=0.0176$. Estas pruebas demuestran que las diferencias encontradas en las edades de salida de la escuela y del primer trabajo fueron diferenciales por cohorte.

En cambio la prueba de Wilcoxon (Breslow) para la igualdad de funciones de supervivencia de la edad mediana a la primera unión conyugal no muestra diferencias estadísticamente significativas por cohorte de mujeres. El estadístico $\chi^{2}$ es de $(1)=1.46$ con $\operatorname{Pr}>\chi^{2}=0.4738$. Lo anterior es congruente con los datos, ya que más que diferencias por cohorte, lo que se observa es una estabilidad en el calendario nupcial en las mujeres que siguieron esta secuencia.

${ }^{16}$ Las edades medianas a la primera unión conyugal se ubicaron en los 18.4, 19.4 y 20.7 años para las cohortes antigua, intermedia y joven de mujeres, respectivamente. 
y 6.5 en la joven; este estrechamiento en el intervalo se debió al aumento de la edad de salida de la escuela y a la estabilidad de la edad mediana a la primera unión conyugal, lo que indica el interés de las mujeres de la cohorte joven por empatar su calendario nupcial con el de las mujeres de las otras cohortes que siguieron la misma trayectoria.

En los hombres también se ve el incremento en el tiempo en las edades medianas de salida de la escuela de la secuencia 3 como parte del aumento generalizado de la escolaridad. Esta salida se presentó entre los 11.7 y los 16.2 años entre cohortes extremas. ${ }^{17} \mathrm{Al}$ realizar la comparación de su edad mediana de salida de la escuela contra la mediana general ${ }^{18}$ de los varones de cada cohorte se aprecian salidas más tempranas entre quienes siguieron esta trayectoria, hasta de 1.2 años en el caso de la cohorte antigua y de 0.3 años para los de la cohorte joven, cosa que no sucedió en las mujeres.

A pesar de sus precoces edades de salida del sistema escolar, los varones del trayecto 3 no obtuvieron un trabajo estable rápidamente, ya que debieron pasar entre tres y cuatro años para que tuvieran un empleo fijo por más de un año, por lo que o bien permanecieron en el hogar o bien trabajaron por periodos cortos en actividades eventuales, dado su bajo nivel escolar de entre primaria y secundaria y su corta edad, sobre todo en las cohortes más antiguas. Este lapso entre las dos primeras transiciones repercutió en que su edad al primer trabajo fuera mayor en más de un año a la del resto de su cohorte. ${ }^{19}$

En los varones la transición de comenzar a vivir en pareja se postergó hasta pasados los 23 años, cuando posiblemente ya contaban con algún capital para iniciar su propia familia. Nuevamente sus edades medianas fueron mayores a las del resto de su cohorte. ${ }^{20}$ La duración de esta trayectoria se fue acortando al pasar de 13 a 7 años entre co-

${ }^{17}$ La prueba de regresión de Cox para la igualdad de curvas de supervivencia de la salida de la escuela muestra diferencias estadísticamente significativas según cohorte de hombres. El estadístico Wald $\chi^{2}$ es de $(1)=24.08$ con $\operatorname{Pr}>\chi^{2}=0.0000$. La prueba de Wilcoxon (Breslow) para la igualdad de funciones de supervivencia de la edad mediana al primer empleo también muestra diferencias estadísticamente significativas por cohorte de hombres. El estadístico $\chi^{2}$ es de $(1)=24.66$ con $\operatorname{Pr}>\chi^{2}=0.0000$. En tanto la prueba de Wilcoxon (Breslow) para la igualdad de funciones de supervivencia de la edad mediana a la primera unión conyugal no muestra diferencias estadísticamente significativas por cohorte de hombres. El estadístico $\chi^{2}$ es de $(1)=3.45$ con $\operatorname{Pr}>\chi^{2}=0.1780$.

${ }^{18}$ Las edades medianas a la salida de la escuela se situaron en los $12.9,14.5$ y 16.7 años para las cohortes antigua, intermedia y joven de varones, respectivamente.

${ }^{19}$ En los hombres la edad mediana al primer trabajo estable fue de $14.6,16.4$ y 17.1 años para las cohortes antigua, intermedia y joven, respectivamente.

${ }^{20}$ Las edades medianas a la primera unión conyugal fueron 24, 22.6 y 22.6 años para las cohortes antigua, intermedia y joven de hombres, respectivamente. 
hortes, como consecuencia principalmente del aumento de la edad de salida de la escuela.

Las edades de salida del sistema escolar y de ingreso a la fuerza laboral fueron muy similares entre hombres y mujeres en esta trayectoria. Las mayores diferencias se registraron en el calendario nupcial, el cual en todo momento fue mayor para ellos, lo cual coincide con el patrón general de la nupcialidad que ha primado no sólo en México, sino alrededor del mundo.

Pareciera que los varones que se apegaron a este itinerario no lo hicieron siguiendo el ideal social de adquirir un mayor nivel educativo y de desempeñarse laboralmente para luego contraer matrimonio, como supone el enfoque del curso de vida, sino que más bien pudieran haber sido orillados a ello por diversas situaciones que no les permitieron adquirir un mayor capital humano y que simultáneamente pudieron haber influido en su retraso en la obtención de un trabajo estable y en la consolidación de su vida matrimonial. En cambio las mujeres que siguieron esta trayectoria adquirieron un capital humano superior o al menos igual al del resto de su cohorte, a la vez que adelantaron su ingreso a la vida laboral y retardaron su vida conyugal, mostrando un patrón normativo en cuanto al ordenamiento de sus transiciones, a la vez que atípico en cuanto a su temporalidad con respecto a otros grupos de mujeres y a los mismos hombres, por lo que en este caso pareciera que ellas obtuvieron cierta ventaja al seguir esta trayectoria al retardar y adelantar ciertas transiciones. Con ello se observa cómo un mismo conjunto de experiencias puede ser muy diferente en su significado dependiendo de la temporalidad y del intervalo en que éstas se presentan, así como del significado que adquieren para determinada sociedad.

\section{Especialización por sexo: el caso de las trayectorias 4 y 7}

Además de la trayectoria 3, que fue común a hombres y mujeres, se calcularon las edades medianas de otras dos secuencias, las cuales no fueron compartidas por ambos debido precisamente a la especialización por sexo existente en el país a lo largo del siglo pasado. Para las mujeres la segunda trayectoria más importante fue la 4 , tradicional, la cual comienza también con la conclusión de los estudios y concluye con la unión conyugal, sin que hasta los 29 años se presentara su ingreso al mercado laboral, aunque existen indicios de que algunas de ellas realizaron trabajo extradoméstico después de esa edad (gráfica 3). 


\section{GRÁFICA 3}

Trayectoria 4. Edad mediana a la salida de la escuela y a la primera unión conyugal de mujeres por cohorte

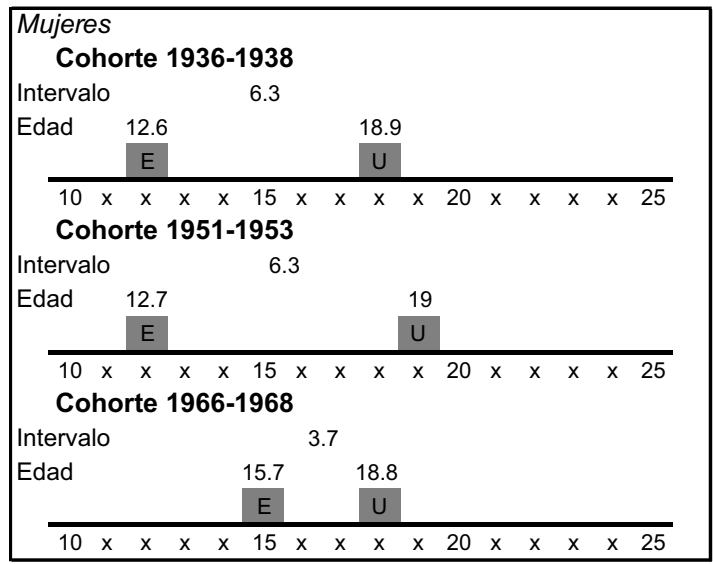

$\mathrm{E}=$ salida de la escuela; $\mathrm{U}=$ primera unión conyugal.

FUENTE: Elaboración propia con base en la submuestra urbana de la Eder, 1998.

En esta trayectoria se aprecian edades medianas de salida del sistema escolar similares a las del total de sus respectivas cohortes, pero menores a las de su contraparte masculina, con un aumento sostenido a lo largo de seis décadas ${ }^{21}$ (véase las notas 13 y 18). Luego de haber salido de la escuela las mujeres de las generaciones más antiguas dejaron transcurrir un tiempo amplio para comenzar a vivir en pareja. Pero este lapso disminuyó drásticamente en la cohorte más joven, que pasó de 6.3 a 3.7 años con motivo del aumento de su edad mediana a la salida de la escuela que esta cohorte presentó. De cualquier forma, a la edad de 19 años el $50 \%$ de las mujeres de cada una de las tres cohortes que siguieron la secuencia 4 ya se había unido conyugalmente, siendo similares, en el caso de las dos cohortes más antiguas, a la edad mediana general del resto de su generación y menor en la joven.

${ }^{21}$ La prueba de regresión de Cox para la igualdad de curvas de supervivencia de la edad mediana de salida de la escuela presenta diferencias estadísticamente significativas según cohorte de mujeres. El estadístico Wald $\chi^{2}$ es de $(1)=13.54$ con $\operatorname{Pr}>\chi^{2}=0.0012$. Esta prueba muestra que las edades cada vez más tardías de salida de la escuela fueron diferenciales por cohorte.

En cambio la prueba de Wilcoxon (Breslow) para la igualdad de funciones de supervivencia de la edad mediana a la primera unión conyugal no muestra diferencias 
Los datos de esta trayectoria son un reflejo de lo que se reporta en el país, ya que se sabe que los hombres tienden a aventajar ligeramente a las mujeres en términos de escolarización pues ellas suelen salir antes de la escuela. Su retiro escolar viene acompañado de mayores responsabilidades en el hogar y en el cuidado de los menores y de las personas de la tercera edad, así como de una baja participación en el mercado laboral (Giorguli, 2002; Castro y Gandini, 2006).

Más que en la trayectoria 3 , los efectos de los mandatos de género en la conformación de la adultez de las mujeres se hacen patentes en esta secuencia, pues se observa justamente la adaptación a las normas más tradicionales que no promueven o incluso desalientan el desarrollo laboral a la vez que impulsan el inicio de la vida nupcial, y con él la reproducción biológica a temprana edad como formas para constituirse como adultas. Se observa que las expresiones normativas que marcan y regulan las formas, los tiempos, los ritmos y los espacios de la nupcialidad y la reproducción incidieron en la vida de estas mujeres en distintos momentos y espacios (Sánchez, 2013), debido a que éstas se casaron jóvenes y ni antes ni después de su unión conyugal realizaron trabajo extradoméstico estable, desalentadas quizá por sus padres y esposos, a la vez que por la responsabilidad de criar a sus hijos y cuidar de su hogar.

Mientras eso sucedía con las mujeres, los varones presentaron como segunda opción la trayectoria alterna a la normativa 7, que inicia con el ingreso a la fuerza laboral, continúa con la salida de la escuela y concluye con la unión conyugal (gráfica 4). La edad mediana a la que los varones comenzaron a trabajar varió entre los 12 y los 14 años. ${ }^{22}$ Estas edades son mucho más tempranas que la edad mediana general de esta transición de sus respectivas cohortes (véase la nota 19), con diferencias que van de entre dos y cuatro años. Estos hombres debieron

estadísticamente significativas por cohorte de mujeres. El estadístico $\chi^{2}$ es de $(1)=0.46$ con $\operatorname{Pr}>\chi^{2}=0.7934$. Lo anterior concuerda con los datos, pues más que diferencias por cohorte, lo que se observa es una estabilidad en el calendario nupcial en las mujeres que siguieron esta secuencia.

${ }^{22}$ La prueba de Wilcoxon (Breslow) para la igualdad de funciones de supervivencia de la edad mediana al primer empleo no muestra diferencias estadísticamente significativas por cohorte de hombres. El estadístico $\chi^{2}$ es de $(1)=1.35$ con $\operatorname{Pr}>\chi^{2}=0.5095$. La prueba de regresión de Cox para la igualdad de curvas de supervivencia de la salida de la escuela no muestra diferencias estadísticamente significativas según cohorte de hombres. El estadístico Wald $\chi^{2}$ es de $(1)=2.80$ con $\operatorname{Pr}>\chi^{2}=0$. 2463. Similares resultados presenta la prueba de Wilcoxon (Breslow) para la igualdad de funciones de supervivencia de la edad mediana a la primera unión conyugal por cohorte de hombres. El estadístico $\chi^{2}$ es de $(1)=0.05$ con $\operatorname{Pr}>\chi^{2}=0.9749$. 


\section{GRÁFICA 4}

Trayectoria 7. Edad mediana a la entrada al primer trabajo, a la salida de la escuela y a la primera unión conyugal de varones por cohorte

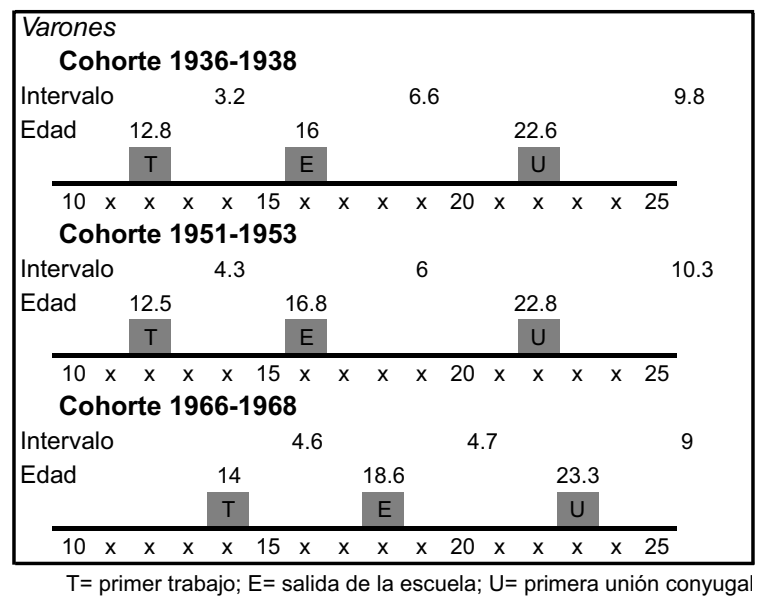

FUENTE: Elaboración propia con base en la submuestra urbana de la Eder, 1998.

tener un mayor interés o necesidad por entrar al mercado laboral, ya sea para contribuir a la economía de su hogar o para financiarse sus estudios.

La última explicación es factible debido a que una vez dentro del mercado de trabajo transcurrieron entre tres y cuatro años para que ellos dejaran la escuela, por lo que es probable que el trabajo les haya facilitado su permanencia en el sistema escolar por más tiempo que el resto de sus compañeros de cohorte al proveerles recursos económicos para ello, según se aprecia al comparar sus edades medianas de salida de la escuela con las totales de cada cohorte, las cuales fueron mucho mayores para los primeros (véase la nota 18).

Coubès y Zenteno (2005) consideran tres posibles explicaciones al hecho de que el incremento de la educación de estas cohortes parece estar asociado a una mayor propensión a iniciar el empleo antes de dejar la escuela: la primera hace referencia al tiempo como un factor que influye en las probabilidades de comenzar a trabajar, por lo que entre mayor tiempo permanezca la persona en la escuela, mayor será el riesgo de que inicie un empleo antes de finalizar los estudios. La siguiente explicación alude a las necesidades económicas de las 
familias, por lo que es posible que éstas requieran del aporte económico de los hijos, aun cuando no hayan terminado la escuela, por lo que éstos deben comenzar a trabajar. La tercera explicación se refiere a que, dada la estabilidad temporal del matrimonio, las personas que no desearan posponerlo tendrían que comenzar a trabajar aun antes de terminar sus estudios para poder casarse a las mismas edades que los demás.

Dichas interpretaciones parecen ser plausibles para la secuencia 7, ya que por una parte se presentó la combinación entre el trabajo y la escuela por un largo tiempo, y por otro lado, una vez fuera del sistema escolar se dio la primera unión alrededor de los 23 años en las tres cohortes.

Como conclusión de este apartado se puede afirmar que el género como estándar normalizador influye en la configuración de determinadas trayectorias y en el calendario de las transiciones que la conforman. Su actuar en la modelación de conductas es diferencial no sólo por sexo, sino también entre hombres y entre mujeres debido al entramado que el género teje con otras jerarquías como las de clase, etnia, raza y generación (Sánchez, 2013).

Así, parecería que algunos grupos sociales tomaron mayores ventajas al seguir determinada secuencia -las mujeres de la trayectoria 3 y los varones de la 7-, en tanto que otros -mujeres de la trayectoria 4 y hombres de la trayectoria 3- muestran ordenamientos más desventajosos, con edades a cada transición que no sólo no se adaptan al modelo normativo enunciado por el curso de vida, sino que difieren negativamente con respecto al resto de sus compañeros de cohorte.

\section{La influencia del estrato socioeconómico en la conformación de trayectorias}

Las trayectorias de vida de la población no sólo son influidas por la cohorte de nacimiento y el género; otros elementos como el estrato socioeconómico ${ }^{23}$ y el lugar de residencia también las afectan. Concre-

${ }^{23}$ En esta sección el número de casos se redujo debido a que no fue posible ubicar a 58 personas por nivel socioeconómico. En las mujeres la población pasó de 688 a 652 casos y en los varones de 578 a 556 casos. Sin embargo los modelos se calcularon utilizando los años-persona, por lo que las observaciones fueron suficientes para estimarlos. Para la construcción del nivel socioeconómico bajo y medio se utilizó la variable de ocupación del padre o de la madre cuando los entrevistados tenían 15 años, debido a que se consideró que la ocupación podría ser un proxy que permitiera situar el nivel de 
tamente la salida de la escuela, como transición que inaugura las trayectorias, es más común a ciertos grupos sociales que a otros. Se sabe que en los estratos bajos ésta suele ser la primera transición, en comparación con el estrato medio alto, siendo las mujeres pobres quienes primeramente dejan el sistema educativo (Oliveira y Mora, 2008). Asimismo, la población rural es quien mayormente la experimenta, además de tener menores índices de asistencia en el nivel adecuado, mayores atrasos y deserción escolar (Giorguli, 2002; Echarri y Pérez Amador, 2007).

Para ahondar en estas desigualdades se presentan dos modelos de historia de eventos; en el primero se examinan la asociación de la cohorte, el sexo, el nivel socioeconómico y la localidad de residencia cuando la persona tenía 15 años, en la probabilidad de comenzar la trayectoria saliendo de la escuela versus iniciarla mediante el ingreso al mercado laboral o nupcial. ${ }^{24}$ Ante la evidencia de otros estudios y los resultados del primer modelo, que muestran que el género es un eje crucial de diferenciación, también se expone otro modelo en donde se prueba que el sexo se interrelaciona con la localidad y con el nivel socioeconómico de origen en la conformación de dichas trayectorias. ${ }^{25}$

Para ajustar estos modelos se eligió la transición de salida de la escuela debido a que el $73 \%$ de las mujeres y el $61 \%$ de los hombres de las tres cohortes habían iniciado de esta manera sus trayectorias. Los modelos muestran en cada edad de la persona la razón de momios (RM) de salir del sistema escolar. ${ }^{26} \mathrm{Si}$ el parámetro es menor a uno en la razón de momios, el efecto de la variable independiente disminuirá el riesgo de iniciar la trayectoria saliendo de la escuela, en tanto que si el efecto es positivo, la razón de momios será mayor a uno.

las personas justo cuando las transiciones que componen estas trayectorias comenzaban a sucederse. Se presentó un menor número de personas ubicadas en el estrato medio en comparación con las del estrato bajo, reflejo de las condiciones de vida que le tocó vivir a los padres de los entrevistados. Se retomó la clasificación de Pacheco (2005) en cinco categorías: profesionistas y directivos, no manual semicalificado, no manual, manual y manual no calificado. Las tres primeras categorías contribuyeron a ubicar tanto a los individuos como a sus familias dentro del nivel medio, en tanto que las dos últimas permitieron ubicarlos en el nivel socioeconómico bajo.

${ }^{24}$ En estos modelos se eliminaron los casos de las personas que no habían tenido oportunidad de asistir al sistema educativo.

${ }^{25}$ Se prefirió presentar el modelo con interacciones, más que estimar dos modelos, uno para hombres y otro para mujeres, ya que justamente se buscaba probar las interacciones del sexo con otras variables, además de que al dividir el número de observaciones los modelos mostraban una menor predicción y estabilidad.

${ }^{26}$ Para mayores referencias sobre las especificaciones del modelo véase Allison, 1984. 


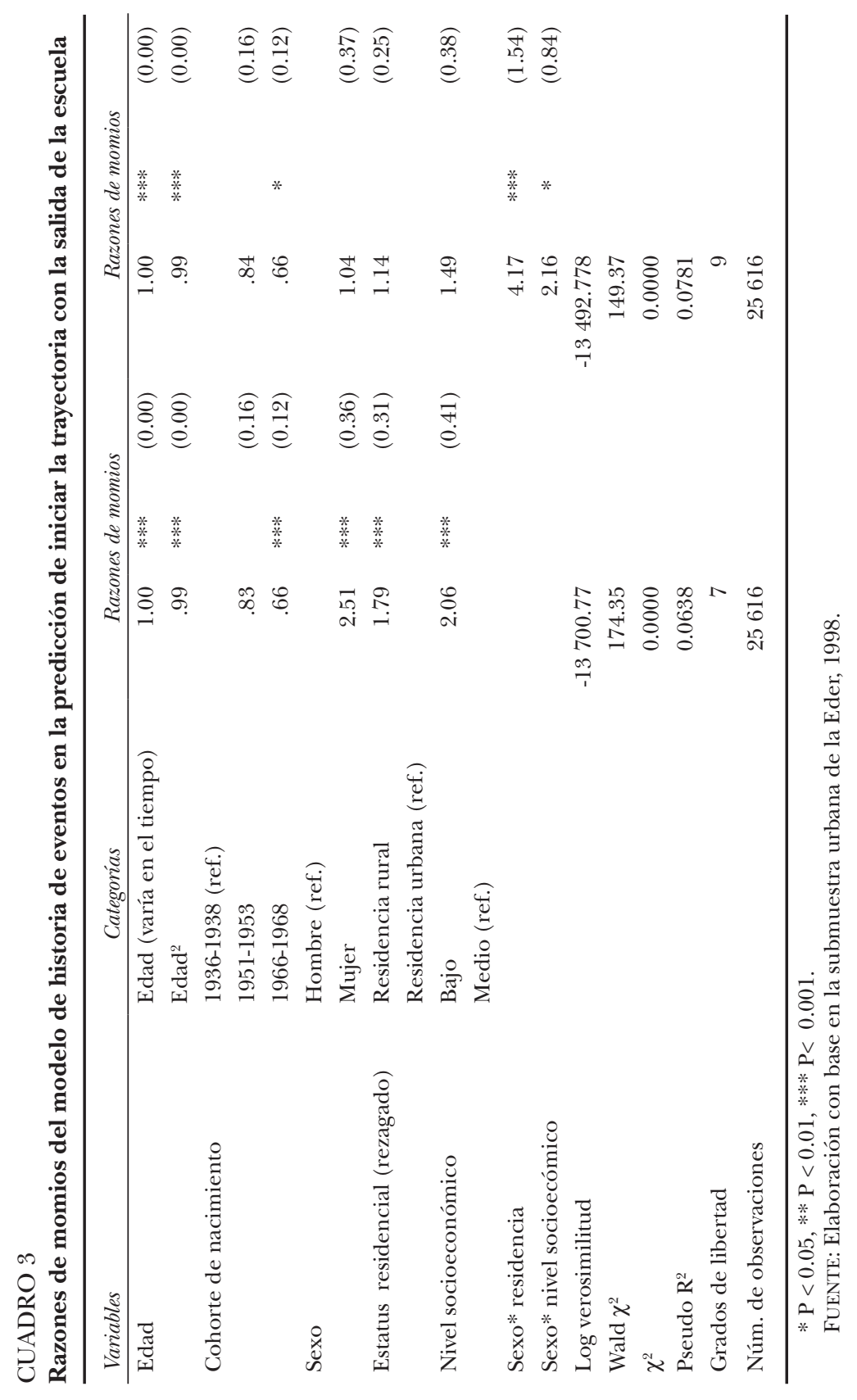


Los modelos del cuadro 3 muestran que con cada año más de vida se incrementa la probabilidad de iniciar la transición a la adultez dejando la escuela. Pero la edad elevada al cuadrado, que también resultó ser significativa, indica que esta relación no es lineal, sino que a partir de cierto momento dicha probabilidad se estaciona o disminuye, seguramente porque al haber salido de la escuela la gran mayoría de las personas queda sólo una muestra selectiva de individuos ubicados en los niveles avanzados de la escolaridad, quienes más que dejar sus estudios, estarían esperando a concluirlos formalmente.

La cohorte joven tuvo una probabilidad menor y estadísticamente significativa de comenzar sus trayectorias con la salida de la escuela. En tanto que las mujeres mostraron una mayor probabilidad de iniciar su adultez dejando la escuela en comparación con los varones, es más factible entonces que ellos la iniciaran trabajando. También las personas que vivían en zonas rurales y las que pertenecían a niveles socioeconómicos bajos cuando tenían 15 años vieron incrementado el riesgo de comenzar sus trayectorias dejando el sistema escolar.

Ante la certeza de que el género hacía una distinción en la manera de comenzar estas trayectorias se realizó un segundo modelo, esta vez con las interacciones de sexo y residencia, y sexo y nivel socioeconómico. En dicho modelo la edad, la edad al cuadrado y la cohorte mantuvieron su significancia. En tanto que ésta desapareció en las variables de estatus residencial y nivel socioeconómico, en cambio las interacciones resultaron significativas, mostrando que las mujeres que en la niñez vivieron en zonas rurales y/o aquellas que pertenecieron al nivel socioeconómico bajo cuando tenían 15 años fueron quienes mayores riesgos tuvieron de comenzar su trayectoria con la transición de salida de la escuela, teniendo como referencia a los hombres urbanos y a los de nivel socioeconómico medio. Estos resultados concuerdan con los enunciados por otros autores (Oliveira y Mora, 2008; Giorguli, 2002; Echarri y Pérez Amador, 2007) y muestran justamente que un tipo de desigualdad, en este caso el género, se interrelaciona con otros como el nivel socioeconómico y el lugar de residencia para configurar ciertos pasos obligados en la transición a la adultez.

\section{Consideraciones finales}

En este artículo se ha tratado de profundizar en el cambio inter e intra cohorte de las trayectorias más comunes de tres cohortes de hombres 
y mujeres mexicanos. La argumentación principal giró en torno a la existencia de trayectorias más típicas a ciertos grupos sociales y a determinados lugares y tiempos, producto de normatividades de género y de su interrelación con las desigualdades socioeconómicas y con los cambios a lo largo de seis décadas. Las preguntas que se pretendió responder se refieren a cuáles serían tales trayectorias, a cómo el género y el nivel socioeconómico habrían dejado su impronta en la conformación de éstas y a qué tan constantes o diferenciales habían sido estas trayectorias en el tiempo.

Los resultados proporcionan evidencias empíricas sobre la variedad de formas que asumen las trayectorias en el inicio de la vida adulta en México, sobre sus diferencias por género y nivel socioeconómico y sobre su variación a través del tiempo; asimismo permiten observar que la no asistencia a la escuela es un elemento que define trayectorias distintas de las de quienes sí asistieron al sistema escolar. En la descripción por cohorte se aprecia que gracias a una política de Estado en materia educativa, las trayectorias sin escolaridad son prácticamente abatidas entre la población más joven, mientras que otras trayectorias alternativas o no tradicionales van tomando presencia al irse incorporando las mujeres a los mercados laborales y al irse posponiendo, en hombres y mujeres, su edad de ingreso a la vida conyugal. Estos datos confirman los hallazgos de otros trabajos en torno a las edades diferenciales de salida de la escuela y de ingreso al mercado laboral y nupcial entre hombres y mujeres (Tuirán, 2001; Giorguli, 2002; Echarri y Pérez Amador, 2007; Oliveira y Mora, 2008).

También muestran que a lo largo de seis décadas se han presentado ciertas continuidades en la conformación del tránsito a la adultez, sobre todo entre la población masculina, pero también que estas trayectorias no siempre son comunes a hombres y mujeres, pues es precisamente en ellas en donde la normatividad de género se manifiesta claramente, permitiendo ver en el orden de las transiciones, en las edades a las que éstas aparecen y en sus intervalos, la síntesis de deseos y elecciones individuales, a la vez que los preceptos normativos sobre lo que hombres y mujeres deben ser y hacer a determinadas edades, el peso de las políticas públicas y las condiciones de posibilidad de la época y de las familias de estas personas.

El género permite no sólo estudiar las desigualdades entre hombres y mujeres, sino también en el interior de cada subpoblación. En el ejercicio sobre las secuencias más representativas para cada género se vislumbró que una misma trayectoria, en este caso la 3 o nor- 
mativa, puede resultar más benéfica si se atrasan algunas transiciones y se adelantan otras, pero también que este beneficio puede estar presente en un grupo y no en otro, en este caso en las mujeres pero no en los hombres. Asimismo, la conformación de trayectorias alternativas (7) o tradicionales (4) indica las distintas formas de acceder a la adultez que ha trazado la sociedad y han recreado las personas que las viven.

En este texto y en otras investigaciones (Solís y Billari, 2003; Coubés y Zenteno, 2005; Echarri y Pérez Amador, 2007; Oliveira y Mora, 2008) se ha reportado que en el inicio de las trayectorias hacia la adultez es posible observar que el género se suele entretejer con el nivel socioeconómico y con el lugar de socialización para inducir a determinadas personas, específicamente a las mujeres de escasos recursos y a las que viven en zonas rurales, a que sean quienes primeramente dejen la escuela en situaciones que no siempre les resultan favorables.

\section{Bibliografía}

Alba, Francisco (1989), La población de México. Evolución y dilemas, México, El Colegio de México.

Alba, Francisco y Joseph E. Potter (1986), "Población y desarrollo en México. Una síntesis de la experiencia reciente”, Estudios Demográficos y Urbanos, vol. 1, núm. 1 (1), pp. 7-37. Disponible en: <http://www.jstor.org/stable/ 40314466>.

Allison, Paul D. (1984), Event History Analysis, Thousand Oakes, SAGE.

Alwin, Duane F. y Ryan J. McCammon (2003), "Generations, Cohorts, and Social Change", en Jeylan T. Mortimer y Michael J. Shanahan (coords.), Handbook of the Life Course, Nueva York, Kluwer Academic / Plenum Publishers, pp. 23-46.

Ariza, Marina (2000), Ya no soy la que dejé atrás... Mujeres migrantes en República Dominicana, México, Instituto de Investigaciones Sociales, UNAM / Plaza y Valdés.

Ariza, Marina y Orlandina de Oliveira (2005), "Unión conyugal e interrupción de la secuencia laboral de las trabajadoras urbanas en México", en MarieLaure Coubès, María Eugenia Zavala de Cosío y René Zenteno (coords.), Cambio demográfico y social en el México del siglo XX. Una perspectiva de historias de vida, México, Cámara de Diputados / EGAP, Tecnológico de Monterrey / El Colegio de la Frontera Norte / Miguel Ángel Porrúa, pp. 429-452.

Billari, Francesco C. (2001), "The Analysis of Early Life Courses: Complex Descriptions of the Transition to Adulthood", Journal of Population Research, vol. 18, núm. 2, pp. 119-142. 
Blanco, Mercedes (2001), "Secuencias laborales y cambio generacional: mujeres de sectores medios en la Ciudad de México", Revista Mexicana de Sociología, vol. 63, núm. 2, pp. 91-111.

Blanco, Mercedes (2002), "Trabajo y familia: entrelazamiento de secuencias vitales”, Estudios Demográficos y Urbanos, vol. 17, núm. 3 (51), pp.447-483. Disponible en: <http://www.jstor.org/stable/40315127>.

Blanco, Mercedes (2011), "El enfoque del curso de vida: orígenes y desarrollo", Revista Latinoamericana de Población, año 5, núm. 8, pp. 5-31.

Blanco, Mercedes y Edith Pacheco (2001), "Secuencias laborales en la Ciudad de México: un acercamiento exploratorio a la articulación de las perspectivas cualitativas y cuantitativas", Revista Latinoamericana de Estudios del Trabajo, año 7, núm. 13, pp. 105-137.

Blanco, Mercedes y Edith Pacheco (2003), "Trabajo y familia desde el enfoque del curso de vida: dos subcohortes de mujeres mexicanas", Papeles de Población, núm. 38, pp. 159-193.

Brugeilles, Carole (2005), "Tendencias de la práctica anticonceptiva en México: tres generaciones de mujeres", en Marie-Laure Coubès, María Eugenia Zavala de Cosío y René Zenteno (coords.), Cambio demográfico y social en el México del siglo XX. Una perspectiva de historias de vida, México, Cámara de Diputados / EGAP, Tecnológico de Monterrey / El Colegio de la Frontera Norte / Miguel Ángel Porrúa, pp. 121-157.

Brugeilles, Carole y Olivia Samuel (2005), "Formación de parejas y vida fecunda en México”, en Marie-Laure Coubès, María Eugenia Zavala de Cosío y René Zenteno (coords.), Cambio demográfico y social en el México del siglo XX. Una perspectiva de historias de vida, México, Cámara de Diputados / EGAP, Tecnológico de Monterrey / El Colegio de la Frontera Norte / Miguel Ángel Porrúa, pp. 453-477.

Butler, Judith (2006), Deshacer el género, Barcelona, Paidós.

Castro Méndez, Nina y Luciana Gandini (2006), "La salida de la escuela y la incorporación al mercado de trabajo de tres cohortes de hombres y mujeres en México", ponencia presentada en el v Congreso Nacional de la Asociación Mexicana de Estudios del Trabajo, "Trabajo y reestructuración: los retos del nuevo siglo", Oaxtepec, México, 17 a 19 de mayo.

Coubés, Marie-Laure (2005), "Movilidad en la trayectoria laboral: transición entre sector formal-informal del empleo", en Marie-Laure Coubès, Ma. Eugenia Zavala y René Zenteno (coords.), Cambio demográfico y social en México en el siglo XX. Una perspectiva de historias de vida, México, Cámara de Diputados / EGAP, Tecnológico de Monterrey / El Colegio de la Frontera Norte / Miguel Ángel Porrúa, pp. 259-282.

Coubès, Marie-Laure, María Eugenia Zavala de Cosío y René Zenteno (2005), "Introducción. La Encuesta Demográfica Retrospectiva”, en Marie-Laure Coubès, María Eugenia Zavala de Cosío y René Zenteno (coords.), Cambio demográfico y social en el México del siglo XX. Una perspectiva de historias de vida, 
México, Cámara de Diputados / EGAP, Tecnológico de Monterrey / El Colegio de la Frontera Norte / Miguel Ángel Porrúa, pp.11-37.

Coubès, Marie-Laure y René Zenteno (2005), "Transición hacia la vida adulta en el contexto mexicano: una discusión a partir del modelo normativo", en Marie-Laure Coubès, María Eugenia Zavala de Cosío y René Zenteno (coords.), Cambio demográfico y social en el México del siglo XX. Una perspectiva de historias de vida, México, Cámara de Diputados / EGAP, Tecnológico de Monterrey / El Colegio de la Frontera Norte / Miguel Ángel Porrúa, pp. $453-477$.

Echarri Cánovas, Carlos Javier (2005), "Las secuencias de corresidencia en la formación de familias”, en Marie-Laure Coubès, María Eugenia Zavala de Cosío y René Zenteno (coords.), Cambio demográfico y social en el México del siglo XX. Una perspectiva de historias de vida, México, Cámara de Diputados / EGAP, Tecnológico de Monterrey / El Colegio de la Frontera Norte / Miguel Ángel Porrúa, pp. 395-427.

Echarri Cánovas, Carlos Javier y Julieta Pérez Amador (2007), "En tránsito hacia la adultez: eventos en el curso de vida de los jóvenes en México", Estudios Demográficos y Urbanos, vol. 22, núm. 1 (64), pp. 43-77. Disponible en: $<$ www.redalyc.org/articulo.oa?id=31222103 $>$.

Edepam (1988), Encuesta sobre Determinantes de la Práctica Anticonceptiva en México, México, Secretaría de Salud.

Eder (1998), Encuesta Demográfica Retrospectiva, México, Instituto Nacional de Estadística, Geografía e Informática.

Elder, Glen H. Jr. (1975), “Age Differentiation and the Life Course”, Annual Review of Sociology, vol. 1, pp. 165-190.

Elder, Glen H. Jr. (1978), "Family History and the Life Course”, en Tamara K. Hareven (coord.), Transitions. The Families and the Life Course in Historical Perspective, Nueva York, Academic Press, pp. 17-64.

Elder, Glen H. Jr. (1985), "Perspectives on the Life Course", en Glen H. Elder, Jr. (coord.), Life Course Dynamics: Trajectories and Transitions, 1968-1980, Ithaca, Cornell University Press, pp. 23-49.

Elder, Glen H. Jr., Monica Kirkpatrick Johnson y Robert Crosnoe (2003), "The Emergence and Development of Life Course Theory", en Jeylan T. Mortimer y Michael J. S. Hanahan (coords.), Handbook of the Life Course, Nueva York, Kluwer Academic / Plenum Publishers, pp. 3-19.

Endifam (2005), Encuesta Nacional sobre la Dinámica de las Familias en México 2005, México, Unidad de Estudios de la Opinión del Instituto de Investigaciones Sociales, UNAM.

García, Brígida (1988), Desarrollo económico y absorción de fuerza de trabajo en México, 1950-1980, México, El Colegio de México.

García, Brígida y Edith Pacheco (2000), "Esposas, hijos e hijas en el mercado de trabajo de la Ciudad de México en 1995”, Estudios Demográficos y Urbanos, vol. 15, núm. 1 (43), pp. 35-63. Disponible en: <www.jstor.org/stable/ $40315021>$. 
García, Brígida y Orlandina de Oliveira (1991), Jefas de hogar y violencia doméstica, México, El Colegio de México / Universidad Nacional Autónoma de México.

Giddens, Anthony (1999), "Runaway World", en <http://news.bbc.co.uk/hi/ english/static/events/reith_99>, Reino Unido, BBC Reith Lectures.

Giorguli, Silvia (2002), "Estructuras familiares y oportunidades educativas de los niños y niñas en México", Estudios Demográficos y Urbanos, vol. 17, núm. 3 (51), pp. 523-546. Disponible en: <www.jstor.org/stable/40315129>.

Giorguli, Silvia (2006), "Deserción escolar, trabajo adolescente y estructuras familiares en México”, en José Luis Lezama y José Morelos (coords.), Población, ciudad y medio ambiente en el México contemporáneo, México, El Colegio de México, pp. 235-274.

Greaves, Cecilia (2011), "La búsqueda de la modernidad", en Dorothy Tanck de Estrada (coord.), La educación en México, México, El Colegio de México, pp. 188-216.

Hogan, Dennis y Marie Aston Nan (1986), "The Transition to Adulthood", Annual Review of Sociology, vol. 12, pp. 109-130.

INEGI (1996), Clasificación Mexicana de Ocupaciones (CMO), tomo I, México, Instituto Nacional de Estadística, Geografía e Informática.

Lagarde, Marcela (1996), Género y feminismo. Desarrollo humano y democracia, Madrid, Horas y Horas.

Mier y Terán, Marta (2012), "Recent Trends and Changes in Mexican Families", trabajo presentado en el congreso anual de la Population Association of America, San Francisco, 3 a 5 de mayo.

Muñoz García, Humberto y Ma. Herlinda Suárez Zozaya (1994), Perfil educativo de la población mexicana, México, INEGI / CRIM, IIS, UNAM.

Oliveira, Orlandina de (1990), "Empleo femenino en México en tiempos de recesión económica: tendencias recientes”, en Neuma Aguiar (coord.), Mujer y crisis. Respuestas ante la recesión, Venezuela, Nueva Sociedad, pp. 31-54.

Oliveira, Orlandina de, Marina Ariza y Marcela Eternod (2001), "La fuerza de trabajo en México: un siglo de cambios”, en José Gómez de León y Cecilia Rabell (coords.), La población en México. Tendencias y perspectivas sociodemográficas hacia el siglo XXI, México, FCE, pp. 873-923.

Oliveira, Orlandina de y Minor Mora Salas (2008), "Desigualdades sociales y transición a la adultez en el México contemporáneo”, Papeles de Población, núm. 57, pp. 117-151.

Pacheco, Edith (2005), "La movilidad ocupacional de los hijos frente a sus padres”, en Marie-Laure Coubès, María Eugenia Zavala de Cosío y René Zenteno (coords.), Cambio demográfico y social en el México del siglo XX. Una perspectiva de historias de vida, México, Cámara de Diputados / EGAP, Tecnológico de Monterrey / El Colegio de la Frontera Norte / Miguel Ángel Porrúa, pp. 227-258. 
Pacheco, Edith y Susan Parker (2001), "Movilidad en el mercado de trabajo urbano: evidencias longitudinales para dos periodos de crisis en México”, Revista Mexicana de Sociología, vol. 63, núm. 2, pp. 3-26.

Pacheco, Edith y René Zenteno (2005), "Medio siglo de incorporación de la mujer a la fuerza de trabajo: cambio social, reestructuración y crisis económica en México", en Marie-Laure Coubès, María Eugenia Zavala de Cosío y René Zenteno (coords.), Cambio demográfico y social en el México del siglo XX. Una perspectiva de historias de vida, México, Cámara de Diputados / EGAP, Tecnológico de Monterrey / El Colegio de la Frontera Norte / Miguel Ángel Porrúa, pp. 191-226.

Parrado, Emilio y René Zenteno (2005), "Medio siglo de incorporación de la mujer a la fuerza de trabajo: cambio social, reestructuración y crisis económica en México", en Marie-Laure Coubès, María Eugenia Zavala de Cosío y René Zenteno (coords.), Cambio demográfico y social en el México del siglo XX. Una perspectiva de historias de vida, México, Cámara de Diputados / EGAP, Tecnológico de Monterrey / El Colegio de la Frontera Norte / Miguel Ángel Porrúa, pp. 65-96.

Quilodrán, Julieta (1996), "Secuencias de vida: un apoyo para la interpretación de los fenómenos demográficos", Estudios Sociológicos, vol. 14, núm. 41, pp. 393-416.

Rendón, Teresa (2003), Trabajo de hombres y trabajo de mujeres en el México del siglo XX, México, CRIM, PUEG, UNAM.

Rendón, Teresa (2004), "El mercado laboral y la división intrafamiliar del trabajo”, en Marina Ariza y Orlandina de Oliveira (coords.), Imágenes de la familia en el cambio de siglo. Universo familiar y procesos de trabajo contemporáneos, México, IIS, UNAM, pp. 49-87.

Samuel, Olivia y Pascal Sebille (2005), "La nupcialidad en movimiento", en Marie-Laure Coubès, María Eugenia Zavala de Cosío y René Zenteno (coords.), Cambio demográfico y social en el México del siglo XX, Tijuana, El Colegio de la Frontera Norte, pp. 41-64.

Sánchez Bringas, Ángeles (2013), "Género, cuerpo y reproducción: desafíos conceptuales y metodológicos en el estudio de las experiencias reproductivas", trabajo presentado en las III Jornadas CINIG de Estudios de Género y Feminismo, La Plata, 25 a 27 de septiembre.

Scott, Joan W. (1996), "El género: una categoría útil para el análisis histórico", en Marta Lamas (comp.), El género: la construcción cultural de la diferencia sexual, México, UnAM / Miguel Ángel Porrúa, pp. 265-302.

Sebille, Pascal (2005), "Primeras etapas de la vida familiar y secuencias migratorias”, en Marie-Laure Coubès, María Eugenia Zavala de Cosío y René Zenteno (coords.), Cambio demográfico y social en el México del siglo XX. Una perspectiva de historias de vida, México, Cámara de Diputados / EGAP, Tecnológico de Monterrey / El Colegio de la Frontera Norte / Miguel Ángel Porrúa, pp. 357-394. 
Solís, Patricio, Cecilia Gayet y Fátima Juárez (2008), "Las transiciones a la vida sexual, a la unión y a la maternidad en México: cambios en el tiempo y estratificación social”, en Susana Lerner e Ivonne Szasz (coords.), Salud reproductiva y condiciones de vida en México, vol. 1, México, El Colegio de México, pp. 397-430.

Solís, Patricio y Francesco C. Billari (2003), "Vidas laborales entre la continuidad y el cambio social: secuencias ocupacionales masculinas en Monterrey, México", Estudios Demográficos y Urbanos, vol. 18, núm. 3 (54), pp. 559-595. Disponible en: <www.jstor.org/stable/40315165>.

Suárez, Leticia (1992), "Secuencias laborales y reproductivas: una comparación entre México y España”, Estudios Demográficos y Urbanos, vol. 7, núm. 2 y 3 (20 y 21), pp. 359-352. Disponible en: <www.jstor.org/stable/40314926>.

Tilly, Charles (2000), La desigualdad persistente, Buenos Aires, Manantial.

Tuirán, Rodolfo (2001), "Dominios institucionales y secuencias de vida en México”, en Beatriz Figueroa Campos (coord.), México diverso y desigual. Enfoques sociodemográficos, vol. 4, México, El Colegio de México / Sociedad Mexicana de Demografía, pp. 207-241.

Zavala de Cosío, María Eugenia (1996), "Políticas de población en México" en Fátima Juárez, Julieta Quilodrán y María Eugenia Zavala de Cosío, Nuevas pautas reproductivas en México, México, El Colegio de México, pp. 199-218.

\section{Acerca de la autora}

Guadalupe Fabiola Pérez Baleón es doctora en Estudios de Población por El Colegio de México; efectuó una estancia de investigación en la Universidad de Brown. Actualmente realiza un posdoctorado en la maestría en Estudios de la Mujer de la Universidad Autónoma Metropolitana con el proyecto "La presencia de las cesáreas en la vida de las mujeres. Análisis de la desigualdad socioeconómica y de género en el proceso reproductivo".

Cuenta con artículos sobre trayectorias reproductivas, así como sobre la salida y el retorno a la escuela y su relación con el ingreso al primer empleo y el mercado matrimonial. Ha sido lectora y directora de varias tesis de maestría y doctorado que versan sobre estas temáticas. 
